\title{
SERIES REPRESENTATION OF POWER FUNCTION
}

\author{
KOLOSOV PETRO
}

\begin{abstract}
In this paper we discuss a problem of generalization of binomial distributed triangle, that is sequence A287326 in OEIS. The main property of A287326 that it returns a perfect cube $n$ as sum of $n$-th row terms over $k$, such that $0 \leq k \leq n-1$ or $1 \leq k \leq n$, by means of its symmetry. In this paper we have derived a similar triangles in order to receive powers $m=5,7$ as row items sum and generalized obtained results in order to receive every odd-powered monomial $n^{2 m+1}, m \geq 0$ as sum of row terms of corresponding triangle. In other words, in this manuscript are found and discussed the polynomials $D_{m}(n, k)$ and $U_{m}(n, k)$, such that, when being summed up over $k$ in some range with respect to $m$ and $n$ returns the monomial $n^{2 m+1}$.
\end{abstract}

\section{Contents}

1. $\quad$ Structure of the manuscript 1

2. Introduction 2

3. Generalization of sequence A287326 6

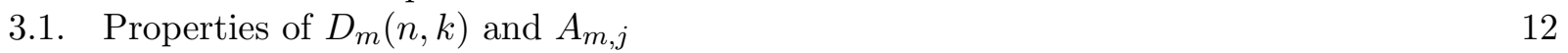

3.2. Example of use of theorem (3.30) 13

4. Another power identity 15

5. Acknowledgements 22

6. Conclusion 23

References 23

7. Application 1. Extended tables of polynomials, consisting $U_{m}(n, k)$ coefficients 24

\section{Structure of the manuscript}

The problem of finding expansions of monomials, binomials, trinomials, etc. is classical and a lot of theorems have been found, the most prominent examples are Binomial Theorem [2], Multinomial theorem, Worpitzky Identity [30], 34], Stirling numbers of second kind and falling factorial identity, [36], etc. In this paper we try to solve the classical problem of finding expansions of monomials. We start from binomial distributed triangle A287326, 11] in OEIS. The main property of A287326 that it returns a perfect cube $n$ as $n$-th row sum, starting from $0, \ldots, n-1$ or from $1, \ldots, n$ by means of its symmetry. Therefore, the following question stated:

- Is there a generalization of A287326 in order to receive monomial $n^{t}, t>3$ as sum of $n$-th row terms of corresponding triangle, where $t$ is natural?

Finding an analogs for $t=5,7$ in section 3, we answer to above questions positively. Could this process be continued for each odd $t=1,3,5,7 \ldots$ similarly? Positive answer to this question is given by theorem (3.30).

Date: August 17, 2018.

Key words and phrases. Series Representation, Power function, monomial, Binomial Theorem, Multinomial theorem, Worpitzky Identity, Stirling numbers of second kind, Faulhabers sum, finite difference, Faulhabers formula, central factorial numbers, binomial coefficients, Binomial distribution, binomial transform, Bernoulli numbers, OEIS, multinomial coefficients. 


\section{INTRODUCTION}

Let describe the derivation of the sequence A287326 in OEIS. Sequence A287326 returns the perfect cube $n$ as row sum over $k, 0 \leq k \leq n-1$, as well as sum over $1 \leq k \leq n$, by means of its symmetry. First, consider a difference table of perfect cubes ([4], eq. 7)

\begin{tabular}{|c|c|c|c|c|}
\hline$n$ & $\Delta^{0}\left(n^{3}\right)$ & $\Delta^{1}\left(n^{3}\right)$ & $\Delta^{2}\left(n^{3}\right)$ & $\Delta^{3}\left(n^{3}\right)$ \\
\hline 0 & 0 & 1 & 6 & 6 \\
\hline 1 & 1 & 7 & 12 & 6 \\
\hline 2 & 8 & 19 & 18 & 6 \\
\hline 3 & 27 & 37 & 24 & 6 \\
\hline 4 & 64 & 61 & 30 & 6 \\
\hline 5 & 125 & 91 & 36 & 6 \\
\hline 6 & 216 & 127 & 42 & 6 \\
\hline 7 & 343 & 169 & 48 & 6 \\
\hline 8 & 512 & 217 & 54 & \\
\hline 9 & 729 & 271 & & \\
\hline 10 & 1000 & & & \\
\hline
\end{tabular}

Table 1: Difference table of perfect cubes $n, 0 \leq n \leq 10$ up to $3^{\text {rd }}$ order.

Reviewing above table, we have noticed that

$$
\begin{aligned}
\Delta\left(0^{3}\right) & =1+6 \cdot 0=6\left(\begin{array}{l}
1 \\
2
\end{array}\right)+\left(\begin{array}{l}
1 \\
0
\end{array}\right) \\
\Delta\left(1^{3}\right) & =1+6 \cdot 0+6 \cdot 1=6\left(\begin{array}{l}
2 \\
2
\end{array}\right)+\left(\begin{array}{l}
2 \\
0
\end{array}\right) \\
\Delta\left(2^{3}\right) & =1+6 \cdot 0+6 \cdot 1+6 \cdot 2=6\left(\begin{array}{l}
3 \\
2
\end{array}\right)+\left(\begin{array}{l}
3 \\
0
\end{array}\right) \\
\Delta\left(3^{3}\right) & =1+6 \cdot 0+6 \cdot 1+6 \cdot 2+6 \cdot 3=6\left(\begin{array}{l}
4 \\
2
\end{array}\right)+\left(\begin{array}{c}
4 \\
0
\end{array}\right) \\
& \vdots \\
\Delta\left(n^{3}\right) & =1+6 \cdot 0+6 \cdot 1+6 \cdot 2+\cdots+6 \cdot n=6\left(\begin{array}{c}
n+1 \\
2
\end{array}\right)+\left(\begin{array}{c}
n+1 \\
0
\end{array}\right)
\end{aligned}
$$

Above difference identity is closely related to Faulhaber's sum of cubes, where $n^{3}=6\left(\begin{array}{c}n+1 \\ 3\end{array}\right)+\left(\begin{array}{c}n+1 \\ 1\end{array}\right)$, see $\left([21]\right.$, p. 9). Note that $\Delta^{2}\left(n^{3}\right)$ could be found similarly using above identity, i.e $\Delta^{2}\left(n^{3}\right)=$ $6\left(\begin{array}{c}n+1 \\ 3-2\end{array}\right)+\left(\begin{array}{c}n+1 \\ 1-2\end{array}\right)$.

Property 2.3. (Generalized finite difference of power using Faulhaber's formula). Consider the identities, ([21], p. 9). For every odd power

$$
\begin{cases}n^{1} & =\left(\begin{array}{c}
n \\
1
\end{array}\right) \\
n^{3} & =6\left(\begin{array}{c}
n+1 \\
3
\end{array}\right)+\left(\begin{array}{c}
n \\
1
\end{array}\right) \\
n^{5} & =120\left(\begin{array}{c}
n+2 \\
5
\end{array}\right)+30\left(\begin{array}{c}
n+1 \\
3
\end{array}\right)+\left(\begin{array}{c}
n \\
1
\end{array}\right) \\
& \vdots \\
n^{2 m-1} & =\sum_{1 \leq k \leq m}(2 k-1) ! T(2 m, 2 k)\left(\begin{array}{c}
n+k-1 \\
2 k-1
\end{array}\right)\end{cases}
$$

The coefficients in these formulas are related to what Riordan [22] has called central factorial numbers of the second kind. In his notation,

$$
x^{m}=\sum_{1 \leq k \leq m} T(m, k) x^{[k]}, x^{[k]}=x\left(x+\frac{k}{2}-1\right)\left(x+\frac{k}{2}-2\right) \cdots\left(x+\frac{k}{2}+1\right)
$$

The coefficients $T(2 m, 2 k)$ are always integers, because the $x^{[k+2]}=x^{[k]}\left(x^{2} / k^{4}\right)$ implies the recurrence

$$
T(2 m+2,2 k)=k^{2} T(2 m, 2 k)+T(2 m, 2 k-2) .
$$


We can find the first order finite difference of odd power as decreasing the variable of corresponding binomial coefficients by 1, for example

$$
\left\{\begin{aligned}
\Delta n^{1} & =\left(\begin{array}{c}
n \\
0
\end{array}\right) \\
\Delta n^{3} & =6\left(\begin{array}{c}
n+1 \\
2
\end{array}\right)+\left(\begin{array}{c}
n \\
0
\end{array}\right) \\
\Delta n^{5} & =120\left(\begin{array}{c}
n+2 \\
4
\end{array}\right)+30\left(\begin{array}{c}
n+1 \\
2
\end{array}\right)+\left(\begin{array}{c}
n \\
0
\end{array}\right) \\
& \vdots \\
\Delta n^{2 m-1} & =\sum_{1 \leq k \leq m}(2 k-1) ! T(2 m, 2 k)\left(\begin{array}{c}
n+k-1 \\
2 k-2
\end{array}\right)
\end{aligned}\right.
$$

Continue similarly, we can express each difference of order $t \geq 1$. The central factorial numbers of the second kind $(2 k-1) ! T(2 m, 2 k)$ in above identities are terms of OEIS sequence $A 303675$ and generated by

$$
(2 k-1) ! T(2 m, 2 k)=\frac{1}{r} \sum_{j=0}^{r}(-1)^{j}\left(\begin{array}{c}
2 r \\
j
\end{array}\right)(r-j)^{2 n}=: V_{m, k},
$$

where $r=n-k+1$, this formula was provided by Peter Luschny in [27]. Repeated sums are equally easy, in Knuth's notation (see [21], p. 10)

$$
\Sigma^{r} n^{2 m+1}=\sum_{1 \leq k \leq m} V_{m, k}\left(\begin{array}{c}
n+k+r \\
2 k-1+r
\end{array}\right)
$$

Therefore, reviewing the difference as inverse operator to summation for every odd $t>0$ and $m \geq 0$, we have identity

$$
\Delta^{r} n^{2 m+1}=\sum_{1 \leq k \leq m} V_{m, k}\left(\begin{array}{c}
n+k-r \\
2 k-1-r
\end{array}\right)
$$

By property 2.3 we rewrite the cubes as

$$
n^{3}=\sum_{1 \leq k \leq n} 6\left(\begin{array}{c}
k+1 \\
2
\end{array}\right)+\left(\begin{array}{l}
k \\
0
\end{array}\right)
$$

Rewrite above expression with set every binomial coefficient to be $\left(\begin{array}{c}n+1 \\ 2\end{array}\right)=1+2+\cdots+(n+1)$, then

$$
n^{3}=(1+6 \cdot 0)+(1+6 \cdot 0+6 \cdot 1)+\cdots+(1+6 \cdot 0+\cdots+6 \cdot(n-1))
$$

Particularizing above expression, we get

$$
n^{3}=n+(n-0) \cdot 6 \cdot 0+(n-1) \cdot 6 \cdot 1+\cdots+(n-(n-1)) \cdot 6 \cdot(n-1)
$$

Provided that $n$ is natural. Now, let apply a compact sigma notation on (2.6), thus

$$
n^{3}=n+\sum_{1 \leq k \leq n} 6 k(n-k)
$$

As sum $\sum_{1 \leq k \leq n} 6 k(n-k)$ consists of $n$ terms, we have right to move $n$ in (2.7) under sigma notation, we get

$$
n^{3}=\sum_{1 \leq k \leq n} 6 k(n-k)+1
$$

Property 2.9. (Proof of symmetry). Let be a sets $A(n):=\{1,2, \ldots, n\}, B(n):=\{0,1, \ldots, n\}$, $C(n):=\{0,1, \ldots, n-1\}$, let be expression 2.8 denoted as

$$
M(n, C(n))=\sum_{k \in C(n)} 6 k(n-k)+1
$$


where $n$ is natural-valued variable and $C(n)$ is iteration set of (2.8), then we have equality

$$
M(n, A(n))=M(n, C(n))
$$

Let review and denote expression (2.6) as

$$
U(n, C(n))=n+6 \cdot \sum_{k \in C(n)} k(n-k)
$$

then

$$
U(n, A(n))=U(n, B(n))=U(n, C(n))
$$

Other words, changing of iteration sets of (2.6) and (2.8) by $A(n), B(n), C(n)$ and $A(n), C(n)$, respectively, doesn't change resulting value for each natural $x$.

Proof. Let be a plot $y(n, k)=6 k(n-k)+1, k \in \mathbb{R}, 0 \leq k \leq 10$, given $n=10$

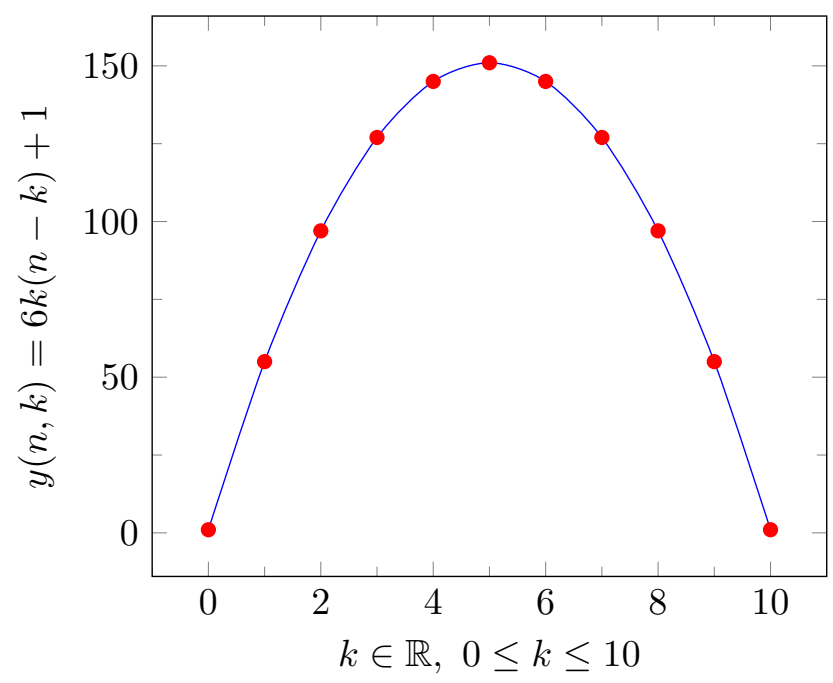

Figure 2. Plot of $6 k(n-k)+1, k \in \mathbb{R}, 0 \leq k \leq n$, where $n=10$.

Obviously, being a parabolic function, it's symmetrical over $\frac{n}{2}$, hence equivalent $M(n, A(n))=$ $M(n, C(n))$ follows. Reviewing (2.6) and denote $u(n, k)=k n-k^{2}$, we can conclude, that $u(n, 0)=$ $u(n, n)=0$, then equality of $U(n, A(n))=U(n, B(n))=U(n, C(n))$ immediately follows. This completes the proof.

Review above property (2.9). Let be an example of triangle built using

Definition 2.12. For every $n \geq 0$

$$
D_{1}(n, k) \stackrel{\text { def }}{=} 6 k(n-k)+1,0 \leq k \leq n
$$

over $n$ from 0 to $n=4$, where $n$ denotes corresponding row and $k$ shows the item of row $n$.

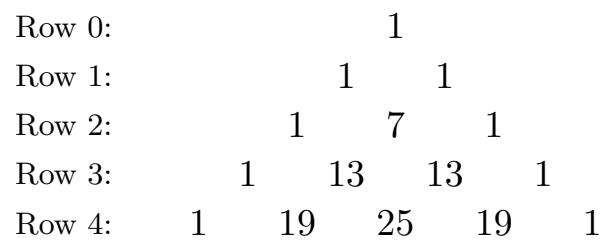

Figure 3. Triangle generated by $D_{1}(n, k)$, sequence A287326 in OEIS, [11. 
Note that $n$-th row sum of Triangle (2.14) over $0 \leq k \leq n-1$ returns perfect cube $n$. We can see that each row with respect to variable $n=0,1,2,3,4, \ldots$, has Binomial distribution of row terms. One could compare Triangle 2.14) with Pascal's triangle [1], [12]

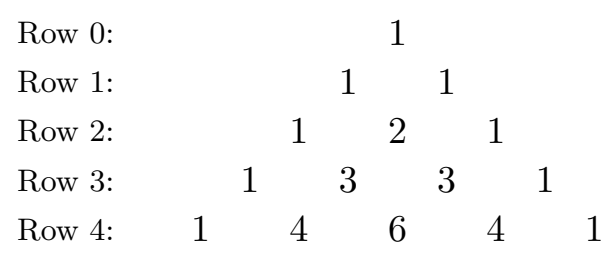

Figure 4. Pascal's triangle, sequence A007318 in OEIS, [1].

Let us approach to show a few properties of triangle (2.14) and $L_{1}(n, k)$.

Properties 2.15. Properties of triangle 2.14).

(1) Summation of $n$-th row of triangle 2.14) over $k$ from 0 to $n-1$ returns perfect cube $n \geq 0$ as follows

$$
n^{3}=\sum_{1 \leq k \leq n} D_{1}(n, k)
$$

(2) First item of each row's number corresponding to central polygonal numbers sequence $a(n)=$ $\frac{n^{2}+n+2}{2}$ (sequence A000124 in OEIS, [13]) returns finite difference of consequent perfect cubes. For example, let be a $k$-th row of triangle 2.14), such that $k=\frac{n^{2}+n+2}{2}, n=0,1,2, \ldots$, then item

$$
\Delta\left(n^{3}\right)=D_{1}\left(\frac{n^{2}+n+2}{2}, 1\right)
$$

(3) Items of (2.14) have Binomial distribution of rows.

(4) Linear recurrence, for every $k$ and $n>0$

$$
2 D_{1}(n, k)=D_{1}(n+1, k)+D_{1}(n-1, k)
$$

This linear recurrence is direct result of second order binomial transform of $D_{1}(n, k)$ by $n$.

(5) Linear recurrence, for each $n>k$

$$
2 D_{1}(n, k)=D_{1}(2 n-k, k)+D_{1}(2 n-k, 0)
$$

(6) From (1.24) for every $n \geq 0$ follows

$$
n^{3}=\sum_{1 \leq k \leq n} D_{1}(n, k)=\sum_{1 \leq k \leq n} D_{1}\left(\frac{k^{2}+k+2}{2}, 1\right)
$$

(7) Triangle 2.14) is symmetric, i.e

$$
D_{1}(n, k)=D_{1}(n, n-k)
$$

Corollary 2.22. Review identity (2.16) in sense of summation of $D_{1}(n, k)$ over some range $W$ with $\max (W)=T$, then (2.16) returns

$$
\sum_{1 \leq k \leq T} D_{1}(n, k)=U_{1}(T, 0) n^{0}-U_{1}(T, 1) n^{1},
$$

where $T=1,2,3, \ldots, \mathbb{N}$. By property 2.9) we rewrite above expression as

$$
\sum_{0 \leq k \leq T-1} D_{1}(n, k)=U_{1}(T-1,0) n^{0}-U_{1}(T-1,1) n^{1}
$$

Below we show a few initial terms of $U_{1}(T-1, j), U_{1}(T, j), j=0,1$ coefficients 


\begin{tabular}{|c|c|c|c|c|}
\hline$T$ & $U_{1}(T-1,0)$ & $U_{1}(T-1,1)$ & $U_{1}(T, 0)$ & $U_{1}(T, 1)$ \\
\hline 1 & 1 & 0 & -5 & 6 \\
2 & -4 & 6 & -28 & 18 \\
3 & -27 & 18 & -81 & 36 \\
4 & -80 & 36 & -176 & 60 \\
5 & -175 & 60 & -325 & 90 \\
6 & -324 & 90 & -540 & 126 \\
7 & -539 & 126 & -833 & 168 \\
8 & -832 & 168 & -1216 & 216 \\
9 & -1215 & 216 & -1701 & 270 \\
10 & -1700 & 270 & -2300 & 330 \\
\hline
\end{tabular}

Table 5. Table of coefficients $U_{1}(T-1,0), U_{1}(T-1,1), U_{1}(T, 0), U_{1}(T, 1)$ given $T=1, \ldots, 10$. Therefore, for every integer $n \geq 1, T=n$,

$$
\forall T=n: n^{3}=\left\{\begin{array}{l}
U_{1}(T, 0) n^{0}-U_{1}(T, 1) n^{1}, \\
U_{1}(T-1,0) n^{0}-U_{1}(T-1,1) n^{1} .
\end{array}\right.
$$

Coefficients $\left|U_{1}(T-1,1)\right|$ are terms of sequence $A 028896$ in OEIS, [23]. Coefficients $\left|U_{1}(T, 0)\right|$ are terms of sequence A275709 in OEIS, [20].

In this section we have derived a binomial distributed triangle (2.14), such that perfect cube $n$ could be found as sum of $n$-th row terms of triangle (2.14) over $k$, in ranges $1 \leq k \leq n$ or $0 \leq k \leq n-1$, where $n$ is natural. Therefore, the follow question is stated:

Question 2.23. Is there a generalization of A287326 in order to receive monomial $n^{t}, t>3$ as sum of $n$-th row terms of corresponding triangle, where $t$ is natural?

\section{Generalization of Sequence A287326}

In order to get analogs of Triangle (2.14) one should solve a system of equations, where unknowns are coefficients of polynomial and variable of polynomial is $k(n-k)$. Triangle (2.14) is generated by polynomial $D_{1}(n, k), n \geq 0,0 \leq k \leq n$, defined by (2.12). Here, let derive a triangle generated by polynomial $D_{2}(n, k), n \geq 0,0 \leq k \leq n$, such that sum of $n$-th row terms over $k$, in ranges $1 \leq k \leq n$ or $0 \leq k \leq n-1$ returns $n^{5}$, where $n$ is natural

Example 3.1. We suspect that $n$-th row of triangle that returns $n^{5}$ as sum of $n$-th row terms over $k$, in $1 \leq k \leq n$ or $0 \leq k \leq n-1$ is generated by

$$
D_{2}(n, k)=A_{2,2}(n-k)^{2} k^{2}+A_{2,1}(n-k)^{1} k^{1}+A_{2,0}(n-k)^{0} k^{0}, n \geq 0,0 \leq k \leq n,
$$

where $A_{2,2}, A_{2,1}, A_{2,0}$ are unknown coefficients. Assume that for every integer $n \geq 0$ holds

$$
n^{5}=\sum_{1 \leq k \leq n} D_{2}(n, k) \text {. }
$$


To determine the coefficients $A_{2,2}, A_{2,1}, A_{2,0}$, in $(3.2)$ let rewrite 3.3$)$ in extended view

$$
\begin{aligned}
& A_{2,2} \sum_{1 \leq k \leq n} k^{2}(n-k)^{2}+A_{2,1} \sum_{1 \leq k \leq n} k(n-k)+\sum_{1 \leq k \leq n} A_{2,0} \\
= & A_{2,2} \sum_{1 \leq k \leq n} k^{2}\left(n^{2}-2 n k+k^{2}\right)+A_{2,1} \sum_{1 \leq k \leq n} k n-k^{2}+\sum_{1 \leq k \leq n} A_{2,0} \\
= & A_{2,2} \sum_{1 \leq k \leq n} k^{2} n^{2}-2 n k^{3}+k^{4}+A_{2,1} \sum_{1 \leq k \leq n} k n-k^{2}+\sum_{1 \leq k \leq n} A_{2,0} \\
= & A_{2,2} n^{2} \sum_{1 \leq k \leq n} k^{2}-2 A_{2,2} n \sum_{1 \leq k \leq n} k^{3}+A_{2,2} \sum_{1 \leq k \leq n} k^{4}+A_{2,1} n \sum_{1 \leq k \leq n} k \\
- & A_{2,1} \sum_{1 \leq k \leq n} k^{2}+\sum_{1 \leq k \leq n} A_{2,0}=n^{5} .
\end{aligned}
$$

Thus, we have received expression containing sums of powers of successive natural numbers, where powers are $\{1,2,3,4\}$. These formulas contain so-called Bernoulli numbers, [14]. For mentioned powers of successive natural numbers formulas are following

$$
\begin{aligned}
\sum_{1 \leq k \leq n} k & =\frac{n^{2}+n}{2}, \\
\sum_{1 \leq k \leq n} k^{2} & =\frac{2 n^{3}+3 n^{2}+n}{6}, \\
\sum_{1 \leq k \leq n} k^{3} & =\frac{n^{4}+2 n^{3}+n^{2}}{4}, \\
\sum_{1 \leq k \leq n} k^{4} & =\frac{6 n^{5}+15 n^{4}+10 n^{3}-n}{30} .
\end{aligned}
$$

Now we substitute above identities to 3.4, respectively,

$$
\begin{aligned}
A_{2,2} n^{2} \frac{2 n^{3}+3 n^{2}+n}{6} & -2 A_{2,2} n \frac{n^{4}+2 n^{3}+n^{2}}{4}+A_{2,2} \frac{6 n^{5}+15 n^{4}+10 n^{3}-n}{30} \\
& +A_{2,1} n \frac{n^{2}+n}{2}-A_{2,1} \frac{2 n^{3}+3 n^{2}+n}{6}+A_{2,0} n
\end{aligned}
$$

Particularizing the elements of above expression and moving them under the common divisor, we get

$$
\frac{A_{2,2} n^{5}-A_{2,2} n+30 A_{2,0}}{30}+A_{2,1} \frac{n^{3}-n}{6}
$$

We have to remember that expression (3.9) is the left side of the input equation (3.3). Therefore,

$$
n^{5}=\frac{A_{2,2} n^{5}-A_{2,2} n+30 A_{2,0}}{30}+A_{2,1} \frac{n^{3}-n}{6}
$$

In order to satisfy 3.10 for each natural $n$, coefficients $A_{2,0}, A_{2,1}, A_{2,2}$ should be a solutions of following system of equations

$$
\begin{cases}\frac{1}{30} A_{2,2} & =1 \\ A_{2,1} & =1 \\ 30 A_{2,0}-A_{2,2} & =0\end{cases}
$$


The only solution of above system is $A_{2,2}=30, A_{2,1}=0, A_{2,0}=1$. Hereby, polynomial $D_{2}(n, k)$ takes the form

$$
D_{2}(n, k)=A_{2,2}(n-k)^{2} k^{2}+A_{2,1}(n-k)^{1} k^{1}+A_{2,0}(n-k)^{0} k^{0}=30 k^{2}(n-k)^{2}+1
$$

And for every natural $n \geq 1$ holds

$$
\begin{aligned}
n^{5} & =\sum_{1 \leq k \leq n} D_{2}(n, k)=\sum_{1 \leq k \leq n} 30 k^{2}(n-k)^{2}+1 \\
& =\sum_{0 \leq k \leq n-1} D_{2}(n, k)=\sum_{0 \leq k \leq n-1} 30 k^{2}(n-k)^{2}+1
\end{aligned}
$$

Let show few initial rows of triangle built by $D_{2}(n, k)$, that is analog of triangle (2.14), which returns monomial $n^{5}$ as sum of $n$-th row terms over $k$, as $1 \leq k \leq n$ or $0 \leq k \leq n-1$, by means of its symmetry

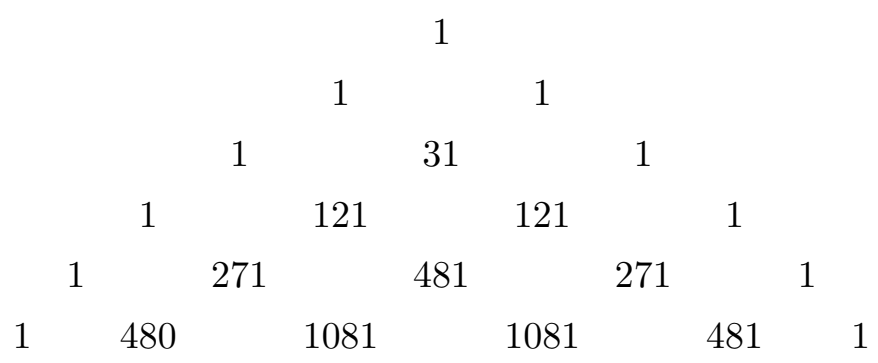

Figure 6. Triangle generated by polynomial $D_{2}(n, k), n \geq 0,0 \leq k \leq n$, sequence A300656 in OEIS, [15].

Similarly, finding the coefficients $A_{3,0}, A_{3,1}, A_{3,2}, A_{3,3}$ in

$$
D_{3}(n, k)=A_{3,3} k^{3}(n-k)^{3}+A_{3,2} k^{2}(n-k)^{2}+A_{3,1} k^{1}(n-k)^{1}+A_{3,0} k^{0}(n-k)^{0},
$$

we get $A_{3,3}=140, A_{3,2}=-14, A_{3,1}=0, A_{3,0}=1$, therefore, for each integer $n \geq 0$ holds

$$
\begin{aligned}
n^{7} & =\sum_{1 \leq k \leq n} D_{3}(n, k)=\sum_{1 \leq k \leq n} 140 k^{3}(n-k)^{3}-14 k^{2}(n-k)^{2}+1 \\
& =\sum_{0 \leq k \leq n-1} D_{3}(n, k)=\sum_{0 \leq k \leq n-1} 140 k^{3}(n-k)^{3}-14 k^{2}(n-k)^{2}+1
\end{aligned}
$$

Below we show a few initial rows of triangle generated by polynomial $D_{3}(n, k), n \geq 0,0 \leq k \leq n$, the analog of triangle (2.14), such that monomial $n^{7}$ could be found as sum of $n$-th row terms over $k$, as $1 \leq k \leq n$ or $0 \leq k \leq n-1$, by means of its symmetry

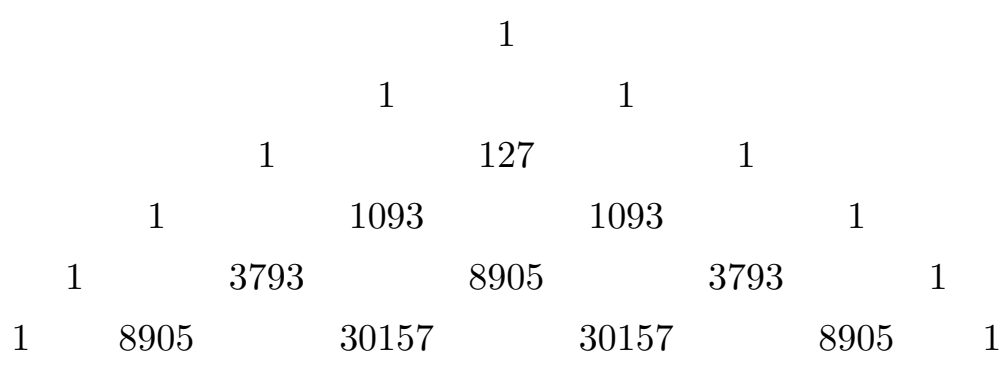

Figure 7. Triangle generated by polynomial $D_{3}(n, k), n \geq 0,0 \leq k \leq n$, sequence A300785 in

OEIS, [16]. 
We assume now that generalization of A287326 holds for odd powers of the form $2 m+1, m=$ $0,1,2, \ldots$, where A287326 is partial case for $m=1$. To generalize our sequences A287326, A300656, A300785 for every odd power $2 m+1, m \geq 0$ one has to review the generating functions of sequences A287326, A300656, A300785 as follows. Let be definition

\section{Definition 3.17.}

$$
\begin{aligned}
D_{m}(n, k) & :=A_{m, m} k^{m}(n-k)^{m}+A_{m, m-1} k^{m-1}(n-k)^{m-1}+\cdots+A_{m, 0} k^{0}(n-k)^{0} \\
& =\sum_{0 \leq j \leq m} A_{m, j} k^{j}(n-k)^{j}
\end{aligned}
$$

where $A_{m, j}, 0 \leq j \leq m$ are unknown coefficients.

And, we assume that

$$
n^{2 m+1}=\sum_{1 \leq k \leq n} D_{m}(n, k)
$$

We want to notice that as we used a compact sigma notation on definition (3.17), i.e we rewrite (3.17) as $\sum_{0 \leq j \leq m} A_{m, j} k^{j}(n-k)^{j}$, thus the sum (3.18) returns indeterminate form of $D_{m}(n, k)=$ $\sum_{0 \leq j \leq m} A_{m, j} k^{j}(n-k)^{j}$ on step $k=n$ as $A_{m, 0}(n-n)^{0} k^{0}$ contains the term $(n-n)^{0}=0^{0}$. Some textbooks leave the quantity $0^{0}$ undefined, because the functions $x^{0}$ and $0^{x}$ have different limiting values when $x$ decreases to 0 . But this is a mistake. We must define

$$
\forall x: x^{0}=1 \text {, }
$$

if the binomial theorem is to be valid when $x=0, y=0$, and/or $x=y$. The binomial theorem is too important to be arbitrarily restricted! By contrast, the function $0^{x}$ is quite unimportant, [31. Note that $D_{m}(n, k)$ is generalization of (2.12) and (3.11). For example, generating functions of sequences A287326, A300656, A300785 are, respectively

$$
\begin{cases}D_{1}(n, k)=1+6 k(n-k), & \text { for } A 287326 \\ D_{2}(n, k)=1-0 k(n-k)+30 k^{2}(n-k)^{2}, & \text { for } A 300656 \\ D_{3}(n, k)=1-14 k(n-k)+0 k^{2}(n-k)^{2}+140 k^{3}(n-k)^{3}, & \text { for } A 300785\end{cases}
$$

Where coefficients $A_{m, j}$, for $m=1,2,3$ are $\left\{A_{1, j}\right\}_{j=0}^{1}=\{1,6\},\left\{A_{2, j}\right\}_{j=0}^{2}=\{1,0,30\},\left\{A_{3, j}\right\}_{j=0}^{3}=$ $\{1,-14,0,140\}$ in definitions of generating functions of A287326, A300656, A300785. To generalize above result in order to receive monomial $n^{2 m+1}$ as $\sum_{1 \leq k \leq n} D_{m}(n, k)=n^{2 m+1}, m=0,1,2, \ldots$ one has to solve the system of equations. Complete set of coefficients $\left\{A_{m, 0}, \ldots, A_{m, m}\right\}$ such that $\sum_{1 \leq k \leq n} D_{m}(n, k)=n^{2 m+1}, m \geq 0$ holds can be found by solving the following system of equations

$$
\left\{\begin{array}{l}
D_{m}(1,0)=1^{2 m+1} \\
D_{m}(2,0)+D_{m}(2,1)=2^{2 m+1} \\
D_{m}(3,0)+D_{m}(3,1)+D_{m}(3,2)=3^{2 m+1} \\
\vdots \\
D_{m}(r, 0)+D_{m}(r, 1)+\cdots+D_{m}(r, r-1)=r^{2 m+1}, r>m
\end{array}\right.
$$

List of solutions $\mathbb{1}^{1}$ of system $\sqrt{3.19}$ is split and assigned to OEIS under the numbers A302971 (numerators of $A_{m, j}$ ) and A304042 (denominators of $A_{m, j}$ ). To reach recurrent formula of $A_{m, j}$,

\footnotetext{
1 solutions_system_2_4.txt - Mathematica code, provides the list of solutions of system 3.19 up to $r=11,24$.
} 
first let fix the unused values $A_{m, j}=0$, for $j<0$ or $j>m$, so we don't need to care about the summation range for $j$, then by expanding $(n-k)^{j}$ and using Faulhaber's formula [7], we get

$$
\begin{aligned}
& \sum_{k=0}^{n-1}(n-k)^{j} k^{j}=\sum_{k=0}^{n-1} \sum_{i}^{\infty}\left(\begin{array}{l}
j \\
i
\end{array}\right) n^{j-i}(-1)^{i} k^{i+j} \\
& =\sum_{i}^{\infty}\left(\begin{array}{c}
j \\
i
\end{array}\right) n^{j-i} \frac{(-1)^{i}}{i+j+1}\left[\sum_{t}^{\infty}\left(\begin{array}{c}
i+j+1 \\
t
\end{array}\right) B_{t} n^{i+j+1-t}-B_{i+j+1}\right] \\
& =\underbrace{\sum_{i, t}^{\infty}\left(\begin{array}{c}
j \\
i
\end{array}\right) \frac{(-1)^{i}}{i+j+1}\left(\begin{array}{c}
i+j+1 \\
t
\end{array}\right) B_{t} n^{2 j+1-t}}_{(\star)}-\underbrace{\sum_{i}^{\infty}\left(\begin{array}{c}
j \\
i
\end{array}\right) \frac{(-1)^{i}}{i+j+1} B_{i+j+1} n^{j-i}}_{(\diamond)}
\end{aligned}
$$

where $B_{t}$ are Bernoulli numbers [14]. Now, we notice that

$$
\sum_{i}^{\infty}\left(\begin{array}{l}
j \\
i
\end{array}\right) \frac{(-1)^{i}}{i+j+1}\left(\begin{array}{c}
i+j+1 \\
t
\end{array}\right)= \begin{cases}\frac{1}{(2 j+1)\left(\begin{array}{c}
2 j \\
j
\end{array}\right)}, & \text { if } t=0 \\
\frac{(-1)^{j}}{t}\left(\begin{array}{c}
j \\
2 j-t+1
\end{array}\right), & \text { if } t>0\end{cases}
$$

In particular, the last sum is zero for $0<t \leq j$. Now we revise the $(\star)$ part of $(3.20)$ according to results of (3.21), thus

$$
\begin{aligned}
\sum_{i, t}^{\infty}\left(\begin{array}{l}
j \\
i
\end{array}\right) \frac{(-1)^{i}}{i+j+1}\left(\begin{array}{c}
i+j+1 \\
t
\end{array}\right) B_{t} n^{2 j+1-t} & =\frac{1}{(2 j+1)\left(\begin{array}{c}
2 j \\
j
\end{array}\right)} \\
& +\sum_{t>0} \frac{(-1)^{j}}{t}\left(\begin{array}{c}
j \\
2 j-t+1
\end{array}\right) B_{t} n^{2 j+1-t}
\end{aligned}
$$

Therefore, 3.20 takes the form

$$
\begin{aligned}
\sum_{k=0}^{n-1}(n-k)^{j} k^{j} & =\underbrace{\frac{1}{(2 j+1)\left(\begin{array}{c}
2 j \\
j
\end{array}\right)}+\sum_{t>0} \frac{(-1)^{j}}{t}\left(\begin{array}{c}
j \\
2 j-t+1
\end{array}\right) B_{t} n^{2 j+1-t}}_{(\star)} \\
& -\underbrace{\sum_{i}^{\infty}\left(\begin{array}{c}
j \\
i
\end{array}\right) \frac{(-1)^{i}}{i+j+1} B_{i+j+1} n^{j-i}}_{(\diamond)}
\end{aligned}
$$

Now, we keep our attention to 3.22 and we have to remember that if the sum over some variable $i$ contains $\left(\begin{array}{l}j \\ i\end{array}\right)$, then instead of limiting its summation range to $i=0, \ldots, j$, we can let $i=-\infty, \ldots,+\infty$ since $\left(\begin{array}{l}j \\ i\end{array}\right)=0$ for $i$ outside the range $i=0, \ldots, j$ (i.e., when $i<0$ or $i>j$ ). It's much easier to review such sum as summing from $-\infty$ to $+\infty$ (unless specified otherwise), where only a finite number of terms are nonzero, this fact is discussed in [28] as well. To combine or cancel identical terms across the two sums in 3.22 more easily, we introduce $\ell=2 j+1-t$ to $(\star)$ and $\ell=j-i$ to $(\diamond)$, 
respectively, we get

$$
\begin{aligned}
& \sum_{k=0}^{n-1}(n-k)^{j} k^{j}=\frac{1}{(2 j+1)\left(\begin{array}{c}
2 j \\
j
\end{array}\right)} n^{2 j+1}+\sum_{\ell=-\infty}^{\infty} \frac{(-1)^{j}}{2 j+1-\ell}\left(\begin{array}{l}
j \\
\ell
\end{array}\right) B_{2 j+1-\ell} n^{\ell} \\
- & \sum_{\ell=-\infty}^{\infty}\left(\begin{array}{l}
j \\
\ell
\end{array}\right) \frac{(-1)^{j-\ell}}{2 j+1-\ell} B_{2 j+1-\ell} n^{\ell} \\
= & \frac{1}{(2 j+1)\left(\begin{array}{c}
2 j \\
j
\end{array}\right)} n^{2 j+1}+2 \sum_{\text {odd } \ell}^{\infty} \frac{(-1)^{j}}{2 j+1-\ell}\left(\begin{array}{l}
j \\
\ell
\end{array}\right) B_{2 j+1-\ell} n^{\ell} .
\end{aligned}
$$

Now, using the definition of $A_{m, j}$, we obtain the following identity for polynomials in $n$

$$
\begin{aligned}
& \sum_{j}^{\infty} A_{m, j} \frac{1}{(2 j+1)\left(\begin{array}{c}
2 j \\
j
\end{array}\right)} n^{2 j+1}+2 \sum_{j, \text { odd } \ell}^{\infty} A_{m, j}\left(\begin{array}{l}
j \\
\ell
\end{array}\right) \frac{(-1)^{j}}{2 j+1-\ell} B_{2 j+1-\ell} n^{\ell} \\
& \equiv n^{2 m+1} .
\end{aligned}
$$

Taking the coefficient of $n^{2 m+1}$ in above expression, we get $A_{m, m}=(2 m+1)\left(\begin{array}{c}2 m \\ m\end{array}\right)$, and taking the coefficient of $x^{2 d+1}$ for an integer $d$ in the range $m / 2 \leq d<m$ we get $A_{m, d}=0$. Taking the coefficient of $n^{2 d+1}$ in 3.24 for $m / 4 \leq d<m / 2$, we get

$$
A_{m, d} \frac{1}{(2 d+1)\left(\begin{array}{c}
2 d \\
d
\end{array}\right)}+2(2 m+1)\left(\begin{array}{c}
2 m \\
m
\end{array}\right)\left(\begin{array}{c}
m \\
2 d+1
\end{array}\right) \frac{(-1)^{m}}{2 m-2 d} B_{2 m-2 d}=0,
$$

i.e

$$
A_{m, d}=(-1)^{m-1} \frac{(2 m+1) !}{d ! d ! m !(m-2 d-1) !} \frac{1}{m-d} B_{2 m-2 d} .
$$

Continue similarly, we can express $A_{m, j}$ for each integer $j$ in range $m / 2^{s+1} \leq j<m / 2^{s}$ (iterating consecutively $s=1,2, \ldots)$ via previously determined values of $A_{m, d}, d<j$ as follows

$$
A_{m, j}=(2 j+1)\left(\begin{array}{c}
2 j \\
j
\end{array}\right) \sum_{d=2 j+1}^{m} A_{m, d}\left(\begin{array}{c}
d \\
2 j+1
\end{array}\right) \frac{(-1)^{d-1}}{d-j} B_{2 d-2 j} .
$$

The same formula holds also for $m=0$. Note that in above sum $m$ have to be $m \geq 2 j+1$ to return nonzero term $A_{m, j}$.

Definition 3.28. We define here a generating function of sequence of coefficients $A_{m, j}$, such that $\sum_{k=0}^{n-1} D_{m}(n, k)=n^{2 m+1}, n \geq 0, m \geq 0$, where $D_{m}(n, k)$ is defined by 3.17

$$
A_{m, j}:= \begin{cases}0, & \text { if } j<0 \text { or } j>m \\
(2 j+1)\left(\begin{array}{c}
2 j \\
j
\end{array}\right) \sum_{d=2 j+1}^{m} A_{m, d}\left(\begin{array}{c}
d \\
2 j+1
\end{array}\right) \frac{(-1)^{d-1}}{d-j} B_{2 d-2 j}, & \text { if } 0 \leq j<m \\
(2 j+1)\left(\begin{array}{c}
2 j \\
j
\end{array}\right), & \text { if } j=m\end{cases}
$$

Five initial rows of triangle generated by $A_{m, j}, j \geq 0,0 \leq j \leq m$ are

$$
\begin{aligned}
& m=0 \quad 1 \\
& m=1 \quad 1 \quad 6 \\
& \begin{array}{llll}
m=2 & 1 & 0 & 30
\end{array} \\
& \begin{array}{lllll}
m=3 & 1 & -14 & 0 & 140
\end{array} \\
& \begin{array}{llllll}
m=4 & 1 & -120 & 0 & 0 & 630
\end{array} \\
& \begin{array}{lllllll}
m=5 & 1 & -1386 & 660 & 0 & 0 & 2772
\end{array}
\end{aligned}
$$


Figure 8. Triangle generated by $A_{m, j}, j \geq 0,0 \leq j \leq m$, sequences A302971 (numerators of $A_{m, j}$ ) and A304042 (denominators of $A_{m, j}$ ).

Note that starting from row $m \geq 11$ the terms of Triangle 3.29 consist fractional numbers, for example, $A_{11,1}=800361655623,6$. One can find complete list of the numerators and denominators of $A_{m, j}$ in OEIS under the identifiers A302971 and A304042, respectively, see [17, [18. To verify the terms that definition (3.28) produces one should refer to Mathematica code ${ }^{2}$. Hereby, let be theorem

Theorem 3.30. For every non-negative integers $n \geq 0$ and $m \geq 0$ holds

$$
n^{V}= \begin{cases}\sum_{1 \leq k \leq n} D_{m}(n, k)=\sum_{1 \leq k \leq n} \sum_{0 \leq j \leq m} A_{m, j} k^{j}(n-k)^{j}, & \text { for } V=2 m+1 \\ \sum_{1 \leq k \leq n} \frac{1}{n} D_{m}(n, k)=\sum_{1 \leq k \leq n} \frac{1}{n} \sum_{0 \leq j \leq m} A_{m, j} k^{j}(n-k)^{j}, & \text { for } V=2 m,\end{cases}
$$

where $D_{m}(n, k)$ is defined by (3.17).

One can verify results concerning above theorem (3.30) via Mathematica code $\mathrm{s}^{3}$ Therefore, theorem (3.30) answers to the question (2.23) positively, since for every $m \geq 0$ exists a triangle, generated by $D_{m}(n, k), n \geq 0,0 \leq k \leq n$, such that odd power $n^{2 m+1}$ can be reached as sum of $n$-th row of corresponding triangle over $1 \leq k \leq n$ or $0 \leq k \leq n-1$. Sequences A287326, A300656, A300785 are partial cases of theorem (3.30) for $m=1,2,3$, respectively.

3.1. Properties of $D_{m}(n, k)$ and $A_{m, j}$. Here we show a few properties of definition $D_{m}(n, k)$, some of them correlates with properties of partial case $D_{1}(n, k)$ in 2.15 .

(1) Sum of $A_{m, j}$ over $j$ in range $0 \leq j \leq m$ gives

$$
\sum_{0 \leq j \leq m} A_{m, j}=2^{2 m+1}-1
$$

where $A_{m, j}$ is defined by 3.28 .

(2) Similarly to property (2.21) of particular case $D_{1}(n, k)$, items of $\left\{D_{m}(n, k)\right\}_{k=0}^{n}, m \geq 0, n \geq$ 0 is symmetric, i.e

for all $k: 0 \leq k \leq n$.

$$
D_{m}(n, k)=D_{m}(n, n-k),
$$

(3) By property (2) for every integer $n \geq 0, m \geq 0$ immediately follows

$$
n^{2 m+1}=\sum_{1 \leq k \leq n} D_{m}(n, k)=\sum_{0 \leq k \leq n-1} D_{m}(n, k)
$$

(4) For every $m \geq 0$ the $A_{m, m}$ are terms of the sequence A002457, [19].

(5) For each $m \geq 0$

$$
A_{m, 0}=1
$$

(6) Assume that $n<0$, then theorem (3.30) can be applied as

$$
n^{V}= \begin{cases}\sum_{1 \leq k \leq|n|} D_{m}(n, k), & \text { for } V=2 m+1 \\ \sum_{1 \leq k \leq|n|} \frac{1}{|n|} D_{m}(|n|, k), & \text { for } V=2 m\end{cases}
$$

2 def_2_12.txt - Mathematica code, implementation of definition 3.28, 25.

3expression_2_1.txt. - Mathematica code, implementation of theorem (3.30) [26]. 
Property 3.31. (Linear Recurrence of $D_{m}(n, k)$.) For every integer $n \geq 0$ in $D_{1}(n, k), D_{2}(n, k)$, $D_{3}(n, k)$ hold the recurrent relations

$$
\begin{aligned}
& D_{1}(n+1, k)=2 D_{1}(n, k)-D_{1}(n-1, k) \\
& D_{2}(n+2, k)=3 D_{2}(n+1, k)-3 D_{2}(n, k)+D_{2}(n-1, k) \\
& D_{3}(n+3, k)=4 D_{3}(n+2, k)-6 D_{3}(n+1, k)+4 D_{3}(n, k)-D_{3}(n-1, k)
\end{aligned}
$$

Review the coefficient $D_{m}(n, k)$, which defined by m-order polynomial. It's well known fact that high order finite difference $\Delta^{m+k} P_{m}(x)=0$ for every $x$, where $P_{m}(x)$ is $m$ order polynomial and $k>0$, 8]. Recall Binomial Transform of sequence $a_{n}$. D. E. Knuth [35] has introduced the binomial transform by

$$
\hat{a}_{n}=\sum_{k=0}^{n}\left(\begin{array}{l}
n \\
k
\end{array}\right)(-1)^{k} a_{k}
$$

In particular, $\hat{a}_{n}=\Delta^{n} a_{n}$, therefore, for $D_{m}(n, k)$ we have

$$
\forall t \geq m: \widehat{D}_{m}(n, k)_{t}=\sum_{j \geq 0}\left[(-1)^{j}\left(\begin{array}{c}
t+1 \\
j
\end{array}\right) D_{m}(n+t-j, k)\right] \equiv 0,
$$

hereby, it gives us right to represent recursively every value of $D_{m}(n+r, k), 0 \leq r \leq t+1$ as

$$
(-1)^{r+1}\left(\begin{array}{c}
t+1 \\
r
\end{array}\right) D_{m}(n+t-r, k)=\sum_{j \in \mathbb{Z}_{\geq 0} /\{r\}}(-1)^{j+1}\left(\begin{array}{c}
t+1 \\
j
\end{array}\right) D_{m}(n+t-j, k)
$$

In particular,

$$
D_{m}(n+t, k)=\sum_{j \geq 1}(-1)^{j+1}\left(\begin{array}{c}
t+1 \\
j
\end{array}\right) D_{m}(n+t-j, k)
$$

Hereby, let be theorem

Theorem 3.36. By property (3.31), particularly, from expression (3.35), for every integer $t \geq m$ follows

$$
(n+t)^{2 m+1}=\sum_{k \geq 1} \sum_{j \geq 1}(-1)^{j+1}\left(\begin{array}{c}
t+1 \\
j
\end{array}\right) D_{m}(n+t-j, k)
$$

Proof. Direct consequence of theorem (3.30) and (3.35).

3.2. Example of use of theorem (3.30). In this subsection we show a detailed application of theorem (3.30), In this subsection we highlight the corresponding terms of $A_{m, j}, 0 \leq j \leq m$ and $T(n, k), 1 \leq k \leq n$ with different colors to be more easily to see regularity. Recall existing pattern, that is triangle of coefficients $A_{m, j}$ defined by 3.28

$$
\begin{aligned}
& m=0 \quad 1 \\
& m=1 \quad 1 \quad 6
\end{aligned}
$$

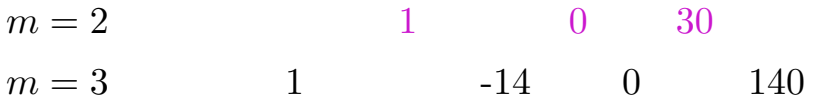

$$
\begin{aligned}
& \begin{array}{llllll}
m=4 & 1 & -120 & 0 & 0 & 630
\end{array} \\
& \begin{array}{lllllll}
m=5 & 1 & -1386 & 660 & 0 & 0 & 2772
\end{array}
\end{aligned}
$$

Figure 9. Triangle generated by $A_{m, j}, m \geq 0,0 \leq j \leq m$. 
By received formula $\sum_{1 \leq k \leq n} \sum_{j \geq 0} D_{m}(n, k)=n^{2 m+1}$ each line of above triangle being multiplied by $T^{j>0}(n, k):=k^{j}(n-k)^{j}$ and summed up to $n$ or $n-1$ over $k$ from 0 or 1 , respectively, will result odd power of $n^{2 m+1}$, depending on the row $A_{m, j}, 0 \leq j \leq m$ is applied. Consider the case $n=3, m=2$, we introduce triangle built using $T(n, k):=k(n-k), n \geq 1,1 \leq k \leq n$ as upper triangular array, let be $1 \leq n \leq 5,1 \leq k \leq n$

\begin{tabular}{ccccc}
$n=1$ & $n=2$ & $n=3$ & $n=4$ & $n=5$ \\
\hline 0 & 1 & 2 & 3 & 4 \\
& 0 & 2 & 4 & 6 \\
& & 0 & 3 & 6 \\
& & & 0 & 4 \\
& & & & 0
\end{tabular}

Figure 10. Upper right triangle generated by $T(n, k)=k(n-k), n \geq 1,1 \leq k \leq n$, sequence A094053, [29] in OEIS.

Example 3.39. Consider theorem (3.30), let be $n=3$ and $m=2$, then

$$
\begin{aligned}
3^{2 \cdot 2+1} & =1 \cdot 2^{0}+0 \cdot 2^{1}+30 \cdot 2^{2} \\
& +1 \cdot \underline{2}^{0}+0 \cdot \underline{2}^{1}+30 \cdot \underline{2}^{2} \\
& +1 \cdot 0^{0}+0 \cdot 0^{1}+30 \cdot 0^{2} \\
& =121+121+1=243
\end{aligned}
$$

Items in above array are terms of the third row of triangle A300656.

Example 3.40. Consider theorem (3.30), let be $n=3$ and $m=3$, then

$$
\begin{aligned}
3^{2 \cdot 3+1} & =1 \cdot 2^{0}-14 \cdot 2^{1}+0 \cdot 2^{2}+140 \cdot 2^{3} \\
& +1 \cdot 2^{0}-14 \cdot 2^{1}+0 \cdot 2^{2}+140 \cdot 2^{3} \\
& +1 \cdot 0^{0}-14 \cdot 0^{1}+0 \cdot 0^{2}+140 \cdot 0^{3} \\
& =1093+1093+1=2187
\end{aligned}
$$

Items in above array are terms of the third row of triangle A300785.

Example 3.41. Consider theorem (3.30), let be $n=4$ and $m=3$, then

$$
\begin{aligned}
4^{2 \cdot 3+1} & =1 \cdot 3^{0}-14 \cdot 3^{1}+0 \cdot 3^{2}+140 \cdot 3^{3} \\
& +1 \cdot 4^{0}-14 \cdot 4^{1}+0 \cdot 4^{2}+140 \cdot 4^{3} \\
& +1 \cdot 3^{0}-14 \cdot 3^{1}+0 \cdot 3^{2}+140 \cdot 3^{3} \\
& +1 \cdot 0^{0}-14 \cdot 0^{1}+0 \cdot 0^{2}+140 \cdot 0^{3} \\
& =3739+8905+3739+1=16384
\end{aligned}
$$

Items in above array are terms of the forth row of triangle A300785. We can perform same result for $4^{2 \cdot 3+1}$ in terms of multiplication of certain matrices,

$$
4^{2 \cdot 3+1}=[1,1,1,1] \cdot\left[\begin{array}{cccc}
3^{0} & 3^{1} & 3^{2} & 3^{3} \\
4^{0} & 4^{1} & 4^{2} & 4^{3} \\
3^{0} & 3^{1} & 3^{2} & 3^{3} \\
0^{0} & 0^{1} & 0^{2} & 0^{3}
\end{array}\right] \cdot\left[\begin{array}{c}
1 \\
-14 \\
0 \\
140
\end{array}\right]
$$


Example 3.42. Consider theorem (3.30), let be $n=5$ and $m=3$, then

$$
\begin{aligned}
5^{2 \cdot 3+1} & =1 \cdot 4^{0}-14 \cdot 4^{1}+0 \cdot 4^{2}+140 \cdot 4^{3} \\
& +1 \cdot 6^{0}-14 \cdot 6^{1}+0 \cdot 6^{2}+140 \cdot 6^{3} \\
& +1 \cdot 6^{0}-14 \cdot 6^{1}+0 \cdot 6^{2}+140 \cdot 6^{3} \\
& +1 \cdot 4^{0}-14 \cdot 4^{1}+0 \cdot 4^{2}+140 \cdot 4^{3} \\
& +1 \cdot 0^{0}-14 \cdot 0^{1}+0 \cdot 0^{2}+140 \cdot 0^{3} \\
& =8905+30157+30157+8905+1=78125
\end{aligned}
$$

Items in above array are terms of the fifth row of triangle A300785.

Example 3.43. Consider theorem (3.30), let be $n=5$ and $m=4$, then

$$
\begin{aligned}
5^{2 \cdot 4+1} & =1 \cdot 4^{0}-120 \cdot 4^{1}+0 \cdot 4^{2}+0 \cdot 4^{3}+630 \cdot 4^{4} \\
& +1 \cdot 6^{0}-120 \cdot 6^{1}+0 \cdot 6^{2}+0 \cdot 6^{3}+630 \cdot 6^{4} \\
& +1 \cdot 6^{0}-120 \cdot 6^{1}+0 \cdot 6^{2}+0 \cdot 6^{3}+630 \cdot 6^{4} \\
& +1 \cdot 4^{0}-120 \cdot 4^{1}+0 \cdot 4^{2}+0 \cdot 4^{3}+630 \cdot 4^{4} \\
& +1 \cdot 0^{0}-120 \cdot 0^{1}+0 \cdot 0^{2}+0 \cdot 0^{3}+630 \cdot 0^{4} \\
& =160801+815761+815761+160801+1=1953125
\end{aligned}
$$

\section{Another POWER IDENTIty}

Review the Corollary (2.2), it says that partial case of theorem (3.30) returns the binomial of the form

$$
\begin{aligned}
\sum_{M \leq k \leq N} D_{1}(n, k) & = \begin{cases}U_{1}(T, 0) n^{0}+U_{1}(T, 1) n^{1}, & \text { if } M=1, N=T \\
U_{1}(T-1,0) n^{0}+U_{1}(T-1,1) n^{1}, & \text { if } M=0, N=T-1\end{cases} \\
& =n^{3}, \text { as } T \rightarrow n .
\end{aligned}
$$

Let extend this idea, as we have complete theorem (3.30), We rewrite theorem (3.30) for odd powers as

$$
\begin{aligned}
\sum_{M \leq k \leq N} D_{m}(n, k) & = \begin{cases}U_{m}(T, 0) n^{0}+\cdots+U_{m}(T, m) n^{m}, & \text { if } M=1, N=T \\
U_{m}(T-1,0) n^{0}+\cdots+U_{m}(T-1, m) n^{m}, & \text { if } M=0, N=T-1\end{cases} \\
& =n^{2 m+1}, \text { as } T=n .
\end{aligned}
$$

Above expression is so-called 'closed form' of the theorem (3.30) for every particular $n$. Below we place a few examples of polynomials $\sum_{0 \leq k \leq m}(-1)^{m-k} U_{m}(T, k) \cdot n^{k}$, where $m=2$,

\begin{tabular}{|c|c|}
\hline$T$ & $U_{2}(T, 0) n^{0}+U_{2}(T, 1) n^{1}+U_{2}(T, 2) n^{2}$ \\
\hline 1 & $31-60 n+30 n^{2}$ \\
2 & $512-540 n+150 n^{2}$ \\
3 & $2943-2160 n+420 n^{2}$ \\
4 & $10624-6000 n+900 n^{2}$ \\
5 & $29375-13500 n+1650 n^{2}$ \\
6 & $68256-26460 n+2730 n^{2}$ \\
7 & $140287-47040 n+4200 n^{2}$ \\
8 & $263168-77760 n+6120 n^{2}$ \\
9 & $459999-121500 n+8550 n^{2}$ \\
10 & $760000-181500 n+11550 n^{2}$ \\
\hline
\end{tabular}


Figure 11. Table of polynomials generated by $\sum_{1 \leq k \leq T} D_{2}(n, k)=n^{5}$ as $T \rightarrow n$, see definition (3.17) for $D_{2}(n, k)$.

Below we show a plots of few first polynomials $\sum_{1<k<T} D_{2}(n, k)$ for $T=1,2,3$ and compare it with corresponding monomial $n^{5}$ by the theorem (3.30),

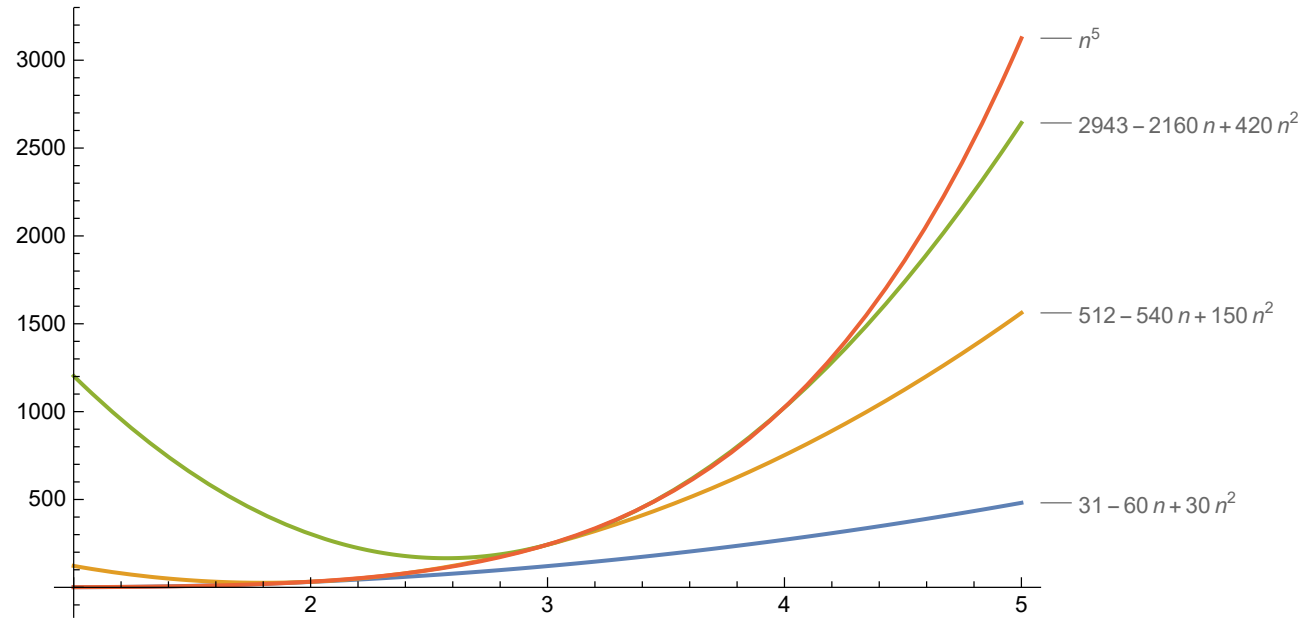

Figure 12. Local approximations of monomial $n^{5}$ by corresponding polynomials $\sum_{1 \leq k \leq T} D_{2}(n, k)$ for $T=1,2,3$, by theorem (3.30).

We can see that monomial $n^{5}$ can be easily approximated in neighborhood of every particular natural $n$. The polynomials from figure (11) can be generated using Mathematica code $\oiint^{4}$ To understand the nature of coefficients $U_{m}(T, k)$, let derive them directly by means of theorem (3.30),

$$
\begin{aligned}
& \sum_{k=1}^{n} \sum_{j=0}^{m} A_{m, j} k^{j}(n-k)^{j}=\sum_{k=1}^{n} A_{m, 0} k^{0}(n-k)^{0}+\cdots+A_{m, m} k^{m}(n-k)^{m} \\
= & \sum_{k=1}^{n} A_{m, 0} k^{0}(n-k)^{0}+\sum_{k=1}^{n} A_{m, 1} k^{1}(n-k)^{1}+\cdots+\sum_{k=1}^{n} A_{m, m} k^{m}(n-k)^{m} \\
= & A_{m, 0} \sum_{k=1}^{n} k^{0}(n-k)^{0}+A_{m, 1} \sum_{k=1}^{n} k^{1}(n-k)^{1}+\cdots+A_{m, m} \sum_{k=1}^{n} k^{m}(n-k)^{m} \\
= & n^{2 m+1} .
\end{aligned}
$$

By the binomial theorem,

$$
\sum_{k=0}^{n-1}(n-k)^{j} k^{j}=\sum_{k=0}^{n-1} \sum_{i=0}\left(\begin{array}{l}
j \\
i
\end{array}\right) n^{j-i}(-1)^{i} k^{j+i}
$$

\footnotetext{
Um(n,k)_coefficients2.txt. - Mathematica code, generates the polynomials from figure 11, 39. If necessary, one could re-define $m \geq 0$ within the code.
} 
We rewrite the main result of $(4.2)$ as

$$
\begin{aligned}
& A_{m, 0} \sum_{k=1}^{n} k^{0}(n-k)^{0}+A_{m, 1} \sum_{k=1}^{n} k^{1}(n-k)^{1}+\cdots+A_{m, m} \sum_{k=1}^{n} k^{m}(n-k)^{m} \\
= & A_{m, 0} \sum_{k=1}^{n} \sum_{i=0}\left(\begin{array}{l}
0 \\
i
\end{array}\right) n^{0-i}(-1)^{i} k^{0+i}+A_{m, 1} \sum_{k=1}^{n} \sum_{i=0}\left(\begin{array}{l}
1 \\
i
\end{array}\right) n^{1-i}(-1)^{i} k^{1+i} \\
+ & A_{m, 2} \sum_{k=1}^{n} \sum_{i=0}\left(\begin{array}{c}
2 \\
i
\end{array}\right) n^{2-i}(-1)^{i} k^{2+i}+A_{m, 3} \sum_{k=1}^{n} \sum_{i=0}\left(\begin{array}{l}
3 \\
i
\end{array}\right) n^{3-i}(-1)^{i} k^{3+i} \\
\vdots & \\
+ & A_{m, m} \sum_{k=1}^{n} \sum_{i=0}\left(\begin{array}{c}
m \\
i
\end{array}\right) n^{m-i}(-1)^{i} k^{m+i}=n^{2 m+1}
\end{aligned}
$$

Rewrite expression 4.3 in extended view

$$
\begin{aligned}
& A_{m, 0} \sum_{k=1}^{n} k^{0}(n-k)^{0}+A_{m, 1} \sum_{k=1}^{n} k^{1}(n-k)^{1}+\cdots+A_{m, m} \sum_{k=1}^{n} k^{m}(n-k)^{m} \\
= & A_{m, 0} \sum_{k=1}^{n}\left[\left(\begin{array}{l}
0 \\
0
\end{array}\right) n^{0}(-1)^{0} k^{0}\right]+A_{m, 1} \sum_{k=1}^{n}\left[\left(\begin{array}{l}
1 \\
0
\end{array}\right) n^{1}(-1)^{0} k^{1}+\left(\begin{array}{l}
1 \\
1
\end{array}\right) n^{0}(-1)^{1} k^{2}\right] \\
+ & A_{m, 2} \sum_{k=1}^{n}\left[\left(\begin{array}{l}
2 \\
0
\end{array}\right) n^{2}(-1)^{0} k^{2}+\left(\begin{array}{l}
2 \\
1
\end{array}\right) n^{1}(-1)^{1} k^{3}+\left(\begin{array}{l}
2 \\
2
\end{array}\right) n^{0}(-1)^{2} k^{4}\right] \\
+ & A_{m, 3} \sum_{k=1}^{n}\left[\left(\begin{array}{l}
3 \\
0
\end{array}\right) n^{3}(-1)^{0} k^{3}+\left(\begin{array}{l}
3 \\
1
\end{array}\right) n^{2}(-1)^{1} k^{4}+\left(\begin{array}{l}
3 \\
2
\end{array}\right) n^{1}(-1)^{2} k^{5}+\left(\begin{array}{l}
3 \\
3
\end{array}\right) n^{0}(-1)^{3} k^{6}\right] \\
\vdots & \quad \\
+ & A_{m, m} \sum_{k=1}^{n}\left[\left(\begin{array}{c}
m \\
0
\end{array}\right) n^{m}(-1)^{0} k^{m}+\left(\begin{array}{c}
m \\
1
\end{array}\right) n^{m-1}(-1)^{1} k^{m+1}+\cdots+\left(\begin{array}{l}
m \\
m
\end{array}\right) n^{0}(-1)^{m} k^{2 m}\right] \\
= & n^{2 m+1} .
\end{aligned}
$$

Let rewrite expression (4.4) again and move sigma notation under the brackets,

$$
\begin{array}{ll}
\text { 4.5) } & n^{2 m+1}=A_{m, 0} \sum_{k=1}^{n} k^{0}(n-k)^{0}+\cdots+A_{m, m} \sum_{k=1}^{n} k^{m}(n-k)^{m} \\
= & A_{m, 0}\left[\sum_{k=1}^{n}\left(\begin{array}{l}
0 \\
0
\end{array}\right) n^{0}(-1)^{0} k^{0}\right]+A_{m, 1}\left[\sum_{k=1}^{n}\left(\begin{array}{l}
1 \\
0
\end{array}\right) n^{1}(-1)^{0} k^{1}+\sum_{k=1}^{n}\left(\begin{array}{l}
1 \\
1
\end{array}\right) n^{0}(-1)^{1} k^{2}\right] \\
+ & A_{m, 2}\left[\sum_{k=1}^{n}\left(\begin{array}{l}
2 \\
0
\end{array}\right) n^{2}(-1)^{0} k^{2}+\sum_{k=1}^{n}\left(\begin{array}{l}
2 \\
1
\end{array}\right) n^{1}(-1)^{1} k^{3}+\sum_{k=1}^{n}\left(\begin{array}{l}
2 \\
2
\end{array}\right) n^{0}(-1)^{2} k^{4}\right] \\
+ & A_{m, 3}\left[\sum_{k=1}^{n}\left(\begin{array}{l}
3 \\
0
\end{array}\right) n^{3}(-1)^{0} k^{3}+\sum_{k=1}^{n}\left(\begin{array}{l}
3 \\
1
\end{array}\right) n^{2}(-1)^{1} k^{4}+\sum_{k=1}^{n}\left(\begin{array}{l}
3 \\
2
\end{array}\right) n^{1}(-1)^{2} k^{5}+\sum_{k=1}^{n}\left(\begin{array}{l}
3 \\
3
\end{array}\right) n^{0}(-1)^{3} k^{6}\right] \\
\vdots & {\left[\sum_{k=1}^{n}\left(\begin{array}{c}
m \\
0
\end{array}\right) n^{m}(-1)^{0} k^{m}+\sum_{k=1}^{n}\left(\begin{array}{c}
m \\
1
\end{array}\right) n^{m-1}(-1)^{1} k^{m+1}+\cdots+\sum_{k=1}^{n}\left(\begin{array}{l}
m \\
m
\end{array}\right) n^{0}(-1)^{m} k^{2 m}\right]}
\end{array}
$$


For example, consider the polynomial $\sum_{k=0}^{m}(-1)^{m-k} U_{m}(n, k) n^{k}$, where $m=3$. We derive below a set of coefficients $U_{3}(n, 0), U_{3}(n, 1), U_{3}(n, 2), U_{3}(n, 3)$

$$
\begin{aligned}
& \sum_{k=0}^{3}(-1)^{3-k} U_{3}(n, k) n^{k} \\
\equiv & A_{3,0}\left[\sum_{k=1}^{n}\left(\begin{array}{l}
0 \\
0
\end{array}\right) n^{0}(-1)^{0} k^{0}\right]+A_{3,1}\left[\sum_{k=1}^{n}\left(\begin{array}{l}
1 \\
0
\end{array}\right) n^{1}(-1)^{0} k^{1}+\sum_{k=1}^{n}\left(\begin{array}{l}
1 \\
1
\end{array}\right) n^{0}(-1)^{1} k^{2}\right] \\
+ & A_{3,2}\left[\sum_{k=1}^{n}\left(\begin{array}{l}
2 \\
0
\end{array}\right) n^{2}(-1)^{0} k^{2}+\sum_{k=1}^{n}\left(\begin{array}{l}
2 \\
1
\end{array}\right) n^{1}(-1)^{1} k^{3}+\sum_{k=1}^{n}\left(\begin{array}{l}
2 \\
2
\end{array}\right) n^{0}(-1)^{2} k^{4}\right] \\
+ & A_{3,3}\left[\sum_{k=1}^{n}\left(\begin{array}{l}
3 \\
0
\end{array}\right) n^{3}(-1)^{0} k^{3}+\sum_{k=1}^{n}\left(\begin{array}{l}
3 \\
1
\end{array}\right) n^{2}(-1)^{1} k^{4}+\sum_{k=1}^{n}\left(\begin{array}{l}
3 \\
2
\end{array}\right) n^{1}(-1)^{2} k^{5}+\sum_{k=1}^{n}\left(\begin{array}{l}
3 \\
3
\end{array}\right) n^{0}(-1)^{3} k^{6}\right] \\
\equiv & n^{7} .
\end{aligned}
$$

The coefficients $U_{3}(n, 0), U_{3}(n, 1), U_{3}(n, 2), U_{3}(n, 3)$ in expression 4.6 are following

$$
\begin{aligned}
U_{3}(n, 0) & =A_{3,0} \sum_{k=1}^{n}\left(\begin{array}{l}
0 \\
0
\end{array}\right)(-1)^{0} k^{0}+A_{3,1} \sum_{k=1}^{n}\left(\begin{array}{l}
1 \\
1
\end{array}\right)(-1)^{1} k^{2} \\
& +A_{3,2} \sum_{k=1}^{n}\left(\begin{array}{l}
2 \\
2
\end{array}\right)(-1)^{2} k^{4}+A_{3,3} \sum_{k=1}^{n}\left(\begin{array}{l}
3 \\
3
\end{array}\right)(-1)^{3} k^{6}, \\
U_{3}(n, 1) & =A_{3,1} \sum_{k=1}^{n}\left(\begin{array}{l}
1 \\
0
\end{array}\right)(-1)^{0} k^{1}+A_{3,2} \sum_{k=1}^{n}\left(\begin{array}{l}
2 \\
1
\end{array}\right)(-1)^{1} k^{3} \\
& +A_{3,3} \sum_{k=1}^{n}\left(\begin{array}{l}
3 \\
2
\end{array}\right)(-1)^{2} k^{5}, \\
U_{3}(n, 2) & =A_{3,2} \sum_{k=1}^{n}\left(\begin{array}{l}
2 \\
0
\end{array}\right)(-1)^{0} k^{2}+A_{3,3} \sum_{k=1}^{n}\left(\begin{array}{l}
3 \\
1
\end{array}\right)(-1)^{1} k^{4} \\
U_{3}(n, 3) & =A_{3,3} \sum_{k=1}^{n}\left(\begin{array}{l}
3 \\
0
\end{array}\right)(-1)^{0} k^{3} .
\end{aligned}
$$

Let rewrite above identities in compact form as,

$$
\begin{aligned}
U_{3}(n, r=0) & =\sum_{t \geq 0}^{3} A_{m, t} \sum_{\ell=1}^{n}\left(\begin{array}{l}
t \\
t
\end{array}\right)(-1)^{t} \ell^{2 t} \\
& =\sum_{t \geq 0}^{3} \sum_{\ell=1}^{n} A_{m, t}\left(\begin{array}{l}
t \\
t
\end{array}\right)(-1)^{t} \ell^{2 t} \\
U_{3}(n, r=1) & =\sum_{t \geq 1}^{3} A_{m, t} \sum_{\ell=1}^{n}\left(\begin{array}{c}
t \\
t-1
\end{array}\right)(-1)^{t-1} \ell^{2 t-1}
\end{aligned}
$$




$$
\begin{aligned}
& =\sum_{t \geq 1}^{3} \sum_{\ell=1}^{n} A_{m, t}\left(\begin{array}{c}
t \\
t-1
\end{array}\right)(-1)^{t-1} \ell^{2 t-1} \\
U_{3}(n, r=2) & =\sum_{t \geq 2}^{3} A_{m, t} \sum_{\ell=1}^{n}\left(\begin{array}{c}
t \\
t-2
\end{array}\right)(-1)^{t-2} \ell^{2 t-2} \\
& =\sum_{t \geq 2}^{3} \sum_{\ell=1}^{n} A_{m, t}\left(\begin{array}{c}
t \\
t-2
\end{array}\right)(-1)^{t-2} \ell^{2 t-2} \\
U_{3}(n, r=3) & =\sum_{t \geq 3}^{3} A_{m, t} \sum_{\ell=1}^{n}\left(\begin{array}{c}
t \\
t-3
\end{array}\right)(-1)^{t-3} \ell^{2 t-3} \\
& =A_{m, 3} \sum_{\ell=1}^{n}\left(\begin{array}{c}
3 \\
t-3
\end{array}\right)(-1)^{0} \ell^{3}
\end{aligned}
$$

Note that above compact forms are depend on $r=0,1,2,3$, as it can be seen by corresponding binomial coefficients, thus we have replaced $k$ by $\ell$, to avoid confusion in notations. Therefore, for every $0 \leq r \leq m$ the $U_{m}(n, r)$ can be defined next way

$$
\begin{aligned}
U_{m}(n, r) & \stackrel{\text { def }}{=} \sum_{t \geq r}^{m} A_{m, t} \sum_{\ell=1}^{n}\left(\begin{array}{c}
t \\
t-r
\end{array}\right)(-1)^{t-r} \ell^{2 t-r} \\
& =\sum_{t \geq r}^{m} \sum_{\ell=1}^{n} A_{m, t}\left(\begin{array}{c}
t \\
t-r
\end{array}\right)(-1)^{t-r} \ell^{2 t-r}
\end{aligned}
$$

Similarly, the coefficients $U_{m}(n-1, r)$ derived just by changing the iteration limits within the sum $\sum_{\ell=1}^{n} A_{m, t}\left(\begin{array}{c}t \\ t-r\end{array}\right)(-1)^{t-r} \ell^{2 t-r}$ in 4.15 from $\ell \in\{1, n\}$ to $\ell \in\{0, n-1\}$

$$
\begin{aligned}
U_{m}(n-1, r) & \stackrel{\text { def }}{=} \sum_{t \geq r}^{m} A_{m, t} \sum_{\ell=0}^{n-1}\left(\begin{array}{c}
t \\
t-r
\end{array}\right)(-1)^{t-r} \ell^{2 t-r} \\
& =\sum_{t \geq r}^{m} \sum_{\ell=0}^{n-1} A_{m, t}\left(\begin{array}{c}
t \\
t-r
\end{array}\right)(-1)^{t-r} \ell^{2 t-r}
\end{aligned}
$$

In above equation (4.16) it must be defined $\ell^{0}=1$ for all $\ell$, see [31]. Mathematica implementations ${ }^{56}$ of expessions 4.15) and 4.16) are available in [41, 42]. Therefore, for every $n \geq 1$, and $m \geq 0$, we have identity

$$
\begin{aligned}
n^{2 m+1} & =\sum_{0 \leq r \leq m}(-1)^{m-r} U_{m}(n, r) \cdot n^{r} \\
& =\sum_{0 \leq r \leq m}(-1)^{m-r} U_{m}(n-1, r) \cdot n^{r}
\end{aligned}
$$

Expressions 4.17), 4.18) are analogs to Faulhaber's odd power identity, see property (2.3) and [21], p. 9. Expressions [4.17, 4.18 could be verified via Mathematica codes ${ }^{7}{ }^{8}$ Here we'd like

\footnotetext{
$5 \operatorname{Um}(n, k)$ coefficients_row_generating.txt. - Mathematica code, implementation of 4.15, prints ten initial rows of coefficients $U_{m}(n, k)$

Um(n-1,k)_coefficients_row_generating.txt. - Mathematica code, implementation of 4.16$)$, prints ten initial rows of coefficients $U_{m}(n-1, k)$

$7 \mathrm{Um}(\mathrm{n}, \mathrm{k}) \_$odd_power_identity.txt - Mathematica code, implementation of 4.17), 37].

¿Um(n,k)_odd_power_identity2.txt - Mathematica code, implementation of 4.18, [38].
} 
to show a few examples of polynomials generated by $\sum_{0 \leq k \leq T-1} D_{2}(n, k)$, see definition (3.17) for $D_{2}(n, k)$

\begin{tabular}{|c|c|}
\hline$T$ & $U_{2}(T-1,0) n^{0}+U_{2}(T-1,1) n^{1}+U_{2}(T-1,2) n^{2}$ \\
\hline 1 & 1 \\
2 & $32-60 n+30 n^{2}$ \\
3 & $513-540 n+150 n^{2}$ \\
4 & $2944-2160 n+420 n^{2}$ \\
5 & $10625-6000 n+900 n^{2}$ \\
6 & $29376-13500 n+1650 n^{2}$ \\
7 & $68257-26460 n+2730 n^{2}$ \\
8 & $140288-47040 n+4200 n^{2}$ \\
9 & $263169-77760 n+6120 n^{2}$ \\
10 & $460000-121500 n+8550 n^{2}$ \\
\hline
\end{tabular}

Figure 13. Table of polynomials generated by $\sum_{0 \leq k \leq T-1} D_{2}(n, k)$.

Below we show a plots of few first polynomials $\sum_{1<k<T-1} D_{2}(n, k)$ for $T=1,2,3,4$ and compare it with corresponding monomial $n^{5}$ by the theorem (3.30)

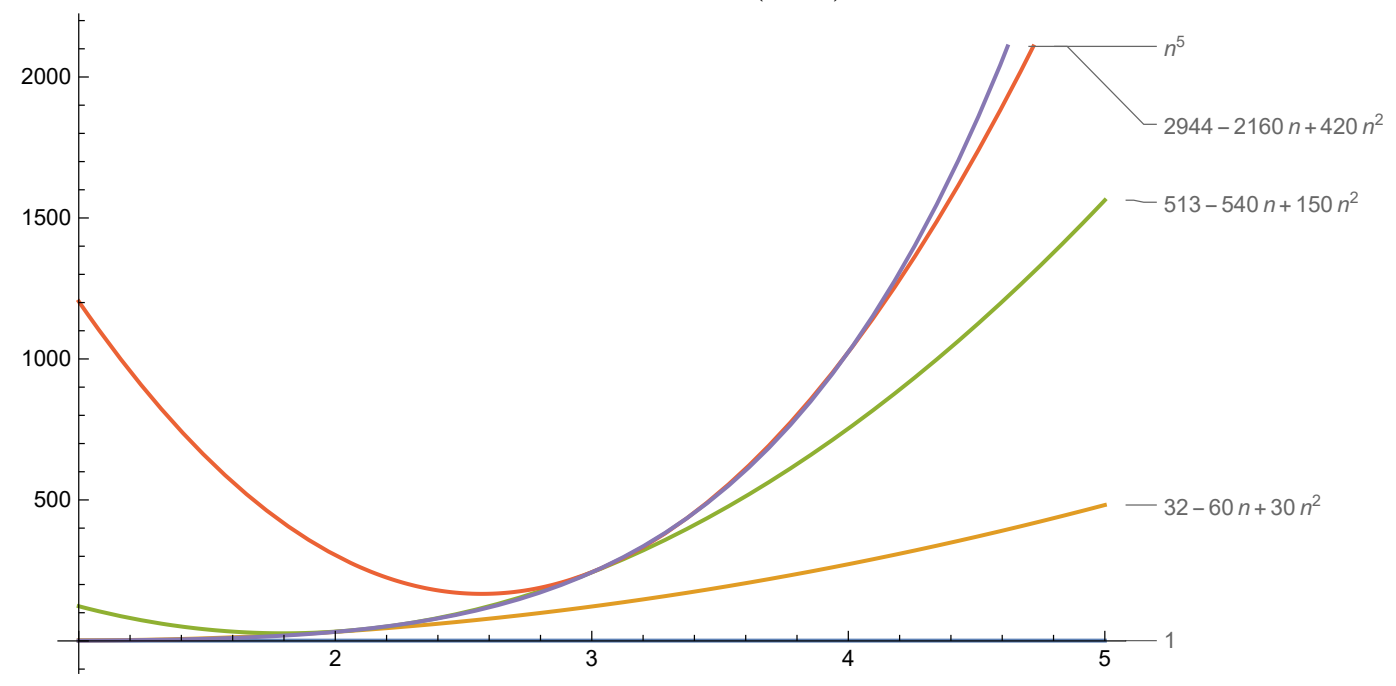

Figure 14. Local approximations of monomial $n^{5}$ by corresponding polynomials $\sum_{1 \leq k \leq T-1} D_{2}(n, k)$ for $T=1,2,3,4$, by theorem (3.30).

The polynomials from figure (13) can be generated using Mathematica code 9 . Additionally, the generalized binomial series could be reached by means of identity

$$
\begin{aligned}
\sum_{M \leq k \leq N} D_{1}(n, k) & = \begin{cases}U_{1}(T, 0) n^{0}+U_{1}(T, 1) n^{1}, & \text { if } M=1, N=T \\
U_{1}(T-1,0) n^{0}+U_{1}(T-1,1) n^{1}, & \text { if } M=0, N=T-1\end{cases} \\
& =n^{3}, \text { as } T \rightarrow n .
\end{aligned}
$$

For instance, for every natural $n$

$$
n^{3}=U_{1}(T, 0) \cdot n^{0}+U_{1}(T, 1) \cdot n^{1}
$$

To reach higher power $m$, let just multiply this identity by $n^{m-3}$,

$$
n^{m}=U_{1}(T, 0) \cdot n^{m-2}+U_{1}(T, 1) \cdot n^{m-3}
$$

\footnotetext{
Um(n,k)_coefficients.txt. - Mathematica code, generates the polynomials from figure 12, 40]. If necessary, one could re-define $m \geq 0$ within the code.
} 
Let rewrite this expression regarding to itself as recursion,

$$
\begin{aligned}
n^{m} & =U_{1}(T, 0) \cdot\left[U_{1}(T, 0) n^{m-4}+U_{1}(T, 1) n^{m-5}\right]+U_{1}(T, 1) \cdot\left[U_{1}(T, 0) n^{m-5}+U_{1}(T, 1) n^{m-6}\right] \\
& =U_{1}(T, 0)^{2} \cdot n^{m-4}-2 \cdot U_{1}(T, 0) \cdot U_{1}(T, 1) n^{m-5}+U_{1}(T, 1)^{2} \cdot n^{m-6}
\end{aligned}
$$

Repeating above process similarly $j \geq 1$ times, we have

$$
\begin{aligned}
n^{m} & =\sum_{k \geq 0}(-1)^{k}\left(\begin{array}{l}
j \\
k
\end{array}\right) U_{1}(T, 0)^{j-k} \cdot U_{1}(T, 1)^{k} \cdot n^{m-2 j-k} \\
& =\sum_{k \geq 0}(-1)^{k}\left(\begin{array}{l}
j \\
k
\end{array}\right) U_{1}(T-1,0)^{j-k} \cdot U_{1}(T-1,1)^{k} \cdot n^{m-2 j-k}
\end{aligned}
$$

We believe we can perform similarly in terms of multinomial coefficients,

$$
\begin{aligned}
n^{m} & =\sum_{k_{1}+k_{2}+\ldots+k_{t}=j}\left(\begin{array}{c}
j \\
k_{1}, k_{2}, \ldots, k_{t}
\end{array}\right) \prod_{\ell=1}^{t}(-1)^{\ell} U_{t}(T, \ell)^{k_{\ell}} \cdot n^{m-(t+1) j-k} \\
& =\sum_{k_{1}+k_{2}+\ldots+k_{t}=j}\left(\begin{array}{c}
j \\
k_{1}, k_{2}, \ldots, k_{t}
\end{array}\right) \prod_{\ell=1}^{t}(-1)^{\ell} U_{t}(T-1, \ell)^{k_{\ell}} \cdot n^{m-(t+1) j-k} .
\end{aligned}
$$

Note that we must set $T=n$ in above identities. Another way to represent odd-powered monomial $n^{2 m+1}, m=0,1,2, \ldots$ is to define a polynomial $\mathcal{U}_{m}(n, k)$. As $\sum_{0 \leq k \leq m}(-1)^{m-k} U_{m}(n, k) \cdot n^{k}=$ $\sum_{0 \leq k \leq m}(-1)^{m-k} U_{m}(n-1, k) \cdot n^{k}$, it follows that

$$
\begin{aligned}
2 n^{2 m+1} & =\sum_{0 \leq k \leq m}(-1)^{m-k} U_{m}(n, k) \cdot n^{k}+\sum_{0 \leq k \leq m}(-1)^{m-k} U_{m}(n-1, k) \cdot n^{k} \\
& =\sum_{0 \leq k \leq m}(-1)^{m-k}\left(U_{m}(n, k)+U_{m}(n-1, k)\right) \cdot n^{k},
\end{aligned}
$$

Let be definition

$$
\mathcal{U}_{m}(n, k) \stackrel{\text { def }}{=} \frac{1}{2}\left[U_{m}(n, k)+U_{m}(n-1, k)\right]
$$

Therefore,

$$
n^{2 m+1}=\sum_{0 \leq k \leq m} \mathcal{U}_{m}(n, k) \cdot n^{k}
$$

Expression 4.23 generates the following polynomials given $m=2$,

\begin{tabular}{|c|c|}
\hline$T$ & $\mathcal{U}_{2}(T, 0) n^{0}+\mathcal{U}_{2}(T, 1) n^{1}+\mathcal{U}_{2}(T, 2) n^{2}$ \\
\hline 1 & $16-30 n+15 n^{2}$ \\
2 & $272-300 n+90 n^{2}$ \\
3 & $1728-1350 n+285 n^{2}$ \\
4 & $6784-4080 n+660 n^{2}$ \\
5 & $20000-9750 n+1275 n^{2}$ \\
6 & $48816-19980 n+2190 n^{2}$ \\
7 & $104272-36750 n+3465 n^{2}$ \\
8 & $201728-62400 n+5160 n^{2}$ \\
9 & $361584-99630 n+7335 n^{2}$ \\
10 & $610000-151500 n+10050 n^{2}$ \\
\hline
\end{tabular}

Figure 15. Table of polynomials generated by $\sum_{0 \leq k \leq m} \mathcal{U}_{m}(T, k) \cdot n^{k}$, where $T=1,2,3, . ., 10$. 
Polynomials from Figure 15 could be generated using Mathematica code $€^{10}$ Let graphically show an approximation of monomial $n^{5}$ by polynomials $\sum_{0 \leq k \leq m} \mathcal{U}_{2}(T, k) \cdot n^{k}$ for $T=1,2,3,4$.

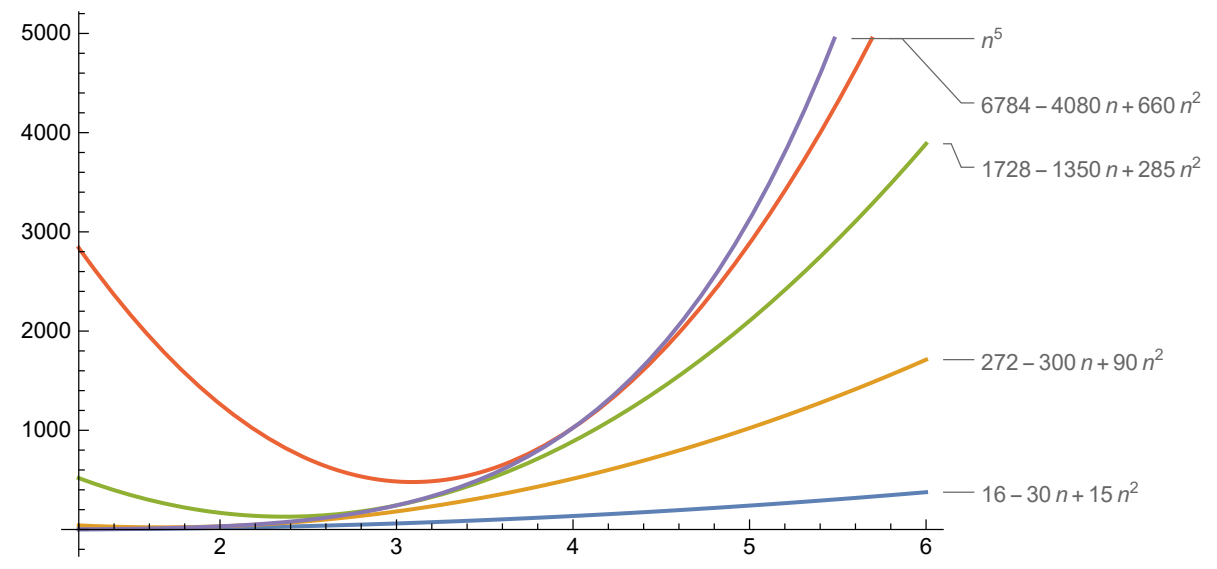

Figure 16. Local approximations of monomial $n^{5}$ by corresponding polynomials

$$
\sum_{0 \leq k \leq m} \mathcal{U}_{2}(T, k) \cdot n^{k} \text { for } T=1,2,3,4 \text {. }
$$

Definition 4.22) of coefficients $\mathcal{U}_{m}(n, k)$ and identity (4.23) could be verified via Mathematica codes ${ }^{11}{ }^{12}$, respectively. Similarly to 4.20, 4.21 we can perform a binomial and multinomial representation of monomial $n^{m}$, where $n, m$ are non-negative integers and $T=n$,

$$
\begin{aligned}
n^{m} & =\sum_{k \geq 0}(-1)^{k}\left(\begin{array}{l}
j \\
k
\end{array}\right) \mathcal{U}_{1}(T, 0)^{j-k} \cdot \mathcal{U}_{1}(T, 1)^{k} \cdot n^{m-2 j-k} \\
& =\sum_{k \geq 0}(-1)^{k}\left(\begin{array}{l}
j \\
k
\end{array}\right) \mathcal{U}_{1}(T-1,0)^{j-k} \cdot \mathcal{U}_{1}(T-1,1)^{k} \cdot n^{m-2 j-k}
\end{aligned}
$$

And

$$
\begin{aligned}
n^{m} & =\sum_{k_{1}+k_{2}+\ldots+k_{t}=j}\left(\begin{array}{c}
j \\
k_{1}, k_{2}, \ldots, k_{t}
\end{array}\right) \prod_{\ell=1}^{t}(-1)^{\ell} \mathcal{U}_{t}(T, \ell)^{k_{\ell}} \cdot n^{m-(t+1) j-k} \\
& =\sum_{k_{1}+k_{2}+\ldots+k_{t}=j}\left(\begin{array}{c}
j \\
k_{1}, k_{2}, \ldots, k_{t}
\end{array}\right) \prod_{\ell=1}^{t}(-1)^{\ell} \mathcal{U}_{t}(T-1, \ell)^{k_{\ell}} \cdot n^{m-(t+1) j-k} .
\end{aligned}
$$

\section{AcKnowledgements}

We would like to thank to Dr. Max Alekseyev (Department of Mathematics and Computational Biology, George Washington University) for sufficient help in deriving of $A_{m, j}$ coefficients, Dr. Hansruedi Widmer for useful comments concerning system of equations (3.19), Dr. Ron Knott (Visiting Fellow, Dept. of Mathematics at University of Surrey) for useful suggestions on writing of this article, and to Mr. Albert Tkaczyk for providing an analogs of triangle (2.14) for powers $m=5,7$, that are sequences A300656, A300785, respectively. Also, we'd like to thank to OEIS editors Michel Marcus, Peter Luschny, Jon E. Schoenfield and others for their patient and faithful volunteer work and for useful comments and suggestions during edition of sequences, concerned with this manuscript. We, also, thank to Tatyana Dryahlova for her help in translating of this manuscript in Russian.

${ }^{10}$ Combined_U_m(n,k)_coefficients_polynomials_gf.txt. - Mathematica code, generates the polynomials from Figure $15,43$.

11 Combined_U_m(n,k)_coefficients_gf.txt. - Mathematica code, verifies the values definition 4.22 produces, 44.

12 Combined_U_m(n,k)_coefficients_odd_power_identity.txt. - Mathematica code, verifies identity 4.23 , [45]. 


\section{Conclusion}

In this paper particular pattern, that is binomial distributed triangle A287326 in OEIS, which shows perfect cube $n$ as sum of row terms over $0 \leq k \leq n-1$ or $1 \leq k \leq n$ is generalized, in this manuscript are found and discussed the polynomials $D_{m}(n, k)$ and $U_{m}(n, k)$, such that, when being summed up over $k$ in some range with respect to $m$ and $n$ returns the monomial $n^{2 m+1}$. As first step, we discussed analogs of A287326 for powers $l=5,7$, sequences A300656, A300785, respectively, then we derived coefficients $A_{m, j}$, such that for every $n \geq 0$ and $m \geq 0$ holds

$$
n^{2 m+1}=\sum_{1 \leq k \leq n} D_{m}(n, k)
$$

where

$$
D_{m}(n, k):=A_{m, m} k^{m}(n-k)^{m}+A_{m, m-1} k^{m-1}(n-k)^{m-1}+\cdots+A_{m, 0} k^{0}(n-k)^{0},
$$

and $A_{m, j}$ is defined by (3.28). Therefore, question question (2.23) is answered positively. Section (3) is totally dedicated to complete and extended derivation of identity $\sum_{1<k<n} D_{m}(n, k)=n^{2 m+1}$. Properties of triangle (2.14) and polynomial $D_{m}(n, k)$ are shown in properties 2.15 and subsection 3.1 respectively. Relation between Faulhaber's sum $\sum n^{m}$ and finite differences of power are shown in 2.3. In section 4 we have generalized the main result of Corollary (2.2) for all odd powers of the form $2 m+1, m=0,1,2, \ldots$ and proven an identities

$$
\begin{aligned}
n^{2 m+1} & =\sum_{0 \leq r \leq m}(-1)^{m-r} U_{m}(n, r) \cdot n^{r} \\
& =\sum_{0 \leq r \leq m}(-1)^{m-r} U_{m}(n-1, r) \cdot n^{r} .
\end{aligned}
$$

\section{REFERENCES}

[1] Conway, J. H. and Guy, R. K. "Pascal's Triangle." In The Book of Numbers. New York: Springer-Verlag, pp. 68-70, 1996.

[2] Abramowitz, M. and Stegun, I. A. (Eds.). Handbook of Mathematical Functions with Formulas, Graphs, and Mathematical Tables, 9th printing. New York: Dover, pp. 10, 1972.

[3] Arfken, G. Mathematical Methods for Physicists, 3rd ed. Orlando, FL: Academic Press, pp. 307-308, 1985.

[4] Weisstein, Eric W. "Finite Difference." From Mathworld

[5] Weisstein, Eric W. "Power.' From Mathworld

[6] Richardson, C. H. An Introduction to the Calculus of Finite Differences. p. 5, 1954.

[7] Johann Faulhaber, Academia Algebræ, Darinnen die miraculosische Inventiones zuden höchsten Cossen weiters continuirt und profitiert werden. Augspurg, bey Johann Ulrich Schönigs, 1631. (Call number QA154.8 F3 1631a f MATH at Stanford University Libraries.), online copy.

[8] Bakhvalov N. S. Numerical Methods: Analysis, Algebra, Ordinary Differential Equations p. 59, 1977. (In russian)

[9] The OEIS Foundation Inc., The On-Line Encyclopedia of Integer Sequences, 1964-present https://oeis.org/

[10] N. J. A. Sloane et al., Entry "Coordination sequence for hexagonal lattice", A008458 in 9], 2002-present.

[11] Petro, Kolosov, et al., Entry "Triangle read by rows: $T(n, k)=6 *(n-k) * k+1$ ", A287326 in [9], 2017.

[12] N. J. A. Sloane and Mira Bernstein et al., "Pascal's Triangle", Entry A007318 in 9], 1994-present.

[13] N. J. A. Sloane. "Central polygonal numbers" Entry A000124 in [9], 1994-present.

[14] Weisstein, Eric W. "Bernoulli Number.' From Mathworld-A Wolfram Web Resource.

[15] Petro, Kolosov, et al., Entry "Triangle read by rows: $T(n, k)=30 *(n-k)^{2} * k^{2}+1$ ", A300656 in [9], 2018.

[16] Petro, Kolosov, et al., Entry "Triangle read by rows: $T(n, k)=140 *(n-k)^{3} * k^{3} *-14 *(n-k) * k+1 ", A 300785$ in 9], 2018.

[17] Petro, Kolosov, et al., Entry "Triangle read by rows: Numeator $\left(A_{m, j}\right), 0 \leq j \leq m, m \geq 0$ ", A302971 in 9 , 2018.

[18] Petro, Kolosov, et al., Entry "Triangle read by rows: Denominator $\left(A_{m, j}\right), 0 \leq j \leq m, m \geq 0 "$, A304042 in 9 , 2018.

[19] N. J. A. Sloane, et al., Entry " $a(n)=(2 n+1) ! / n !^{2} "$, A002457 in 9 .

[20] Joshua Giambalvo, et al., Entry" $a(n)=2 * n^{3}+3 * n^{2} "$, A275709 in 9], 2016. 
[21] Donald E. Knuth., Johann Faulhaber and Sums of Powers, pp. 9-10., arXiv preprint, arXiv:math/9207222v1 [math.CA] 1992.

[22] John Riordan, Combinatorial Identities (New York: John Wiley \& Sons, 1968).

[23] Joe Keane, et al., Entry "6 times triangular numbers: $a(n)=3 * n *(n+1)$ ", A028896 in 9], 1999.

[24] Mathematica code, provides the list of solutions of system $(3.19)$ up to $r=11$ https://kolosovpetro.github.io/mathematica_codes/solutions_system_2_4.txt

[25] Mathematica code, implementation of definition (3.28) https://kolosovpetro.github.io/mathematica_codes/def_2_12.txt

[26] Mathematica code, implementation of theorem (3.30) https://kolosovpetro.github.io/mathematica_codes/expression_2_1.txt

[27] Petro Kolosov, Peter Luschny, Entry "Triangle read by rows: coefficients in the sum of odd powers as expressed by Faulhaber's theorem, $T(n, k)$ for $n \geq 1,1 \leq k \leq n$ ", A303675 in [9], 2018.

[28] Donald E. Knuth., Two notes on notation., pp. 1-2, arXiv preprint, arXiv:math/9205211 [math.HO], 1992.

[29] Reinhard Zumkeller, et al., Entry "Triangle read by rows: $T(n, k)=k(n-k), 1 \leq k \leq n . "$, A094053 in [9], 2004.

[30] Early, Nick., Combinatorics and Representation Theory for Generalized Permutohedra I: Simplicial Plates., p. 3, arXiv preprint, arXiv:1611.06640 [math.CO], 2016.

[31] Ronald Graham, Donald Knuth, and Oren Patashnik (1989-01-05). "Binomial coefficients". Concrete Mathematics (1st ed.). Addison Wesley Longman Publishing Co. p. 162. ISBN 0-201-14236-8, online copy

[32] Anggoro, A.; Liu, E.; and Tulloch, A. "The Rascal Triangle." College Math. J. 41, 393-395, 2010., online copy

[33] Clark Kimberling., Entry "The Rascal Triangle ", A077028 in 9], 2002-present.

[34] Worpitzky, J.. "Studien uber die Bernoullischen und Eulerschen Zahlen.." Journal fur die reine und angewandte Mathematik 94 (1883): 203-232. http://eudml.org/doc/148532.

[35] Donald E. Knuth, The Art of Computer Programming Vol. 3, (1973) Addison-Wesley, Reading, MA.

[36] Agoh, Takashi, and Karl Dilcher. "Generalized convolution identities for Stirling numbers of the second kind." Integers 8.1 (2008): Article-A25, online copy.

[37] Mathematica code, implementation of (4.17), https://kolosovpetro.github.io/mathematica_codes/Um(n,k)_odd_power_identity.txt.

[38] Mathematica code, implementation of (4.18), https://kolosovpetro.github.io/mathematica_codes/Um(n,k)_odd_power_identity2.txt.

[39] Mathematica code, generates the polynomials from figure (11), https://kolosovpetro.github.io/mathematica_codes/Um(n,k)_coefficients.txt.

[40] Mathematica code, generates the polynomials from figure (12), https://kolosovpetro.github.io/mathematica_codes/Um(n,k)_coefficients2.txt.

[41] Mathematica code, implementation of (4.15), prints ten initial rows of coefficients $U_{m}(n, k)$, https://kolosovpetro.github.io/mathematica_codes/Um(n,k)_coefficients_row_generating.txt.

[42] Mathematica code, implementation of (4.16), prints ten initial rows of coefficients $U_{m}(n-1, k)$, https://kolosovpetro.github.io/mathematica_codes/Um(n-1,k)_coefficients_row_generating.txt.

[43] Mathematica code, generates the polynomials from Figure (15), https://kolosovpetro.github.io/mathematica_codes/Combined_U_m(n,k)_coefficients_polynomials_gf.txt.

[44] Mathematica code, verifies the values definition $(4.22)$ produces, https://kolosovpetro.github.io/mathematica_codes/Combined_U_m(n,k)_coefficients_gf.txt.

[45] Mathematica code, verifies identity (4.23), https://kolosovpetro.github.io/mathematica_codes/Combined_U_m(n,k)_coefficients_odd_power_identity.txt.

\section{Application 1. Extended tables of polynomials, Consisting $U_{m}(n, k)$ Coefficients}

In this application we attach an extended table, consisting of polynomials, generated by $\sum_{1 \leq k \leq T} D_{m}(n, k)$ and $\sum_{0 \leq k \leq T-1} D_{m}(n, k)$ for various $t$ and $m$. We begin from cases $m=2,3,4$ given generating function $\sum_{1 \leq k \leq T} D_{m}(n, k)$ and continue similarly with examples for $\sum_{0<k<T-1} D_{m}(n, k)$. The following tables could be generated using Mathematica code Um(n,k)_coefficients2.txt. Here we begin to show our tables for $m=1,2,3,4$ and $T=1,2, \ldots, 40$

\begin{tabular}{|c|c|}
\hline$t$ & Polynomial $(n)$ \\
\hline 1 & $31-60 n+30 n^{2}$ \\
2 & $512-540 n+150 n^{2}$ \\
3 & $2943-2160 n+420 n^{2}$ \\
4 & $10624-6000 n+900 n^{2}$ \\
\hline
\end{tabular}




\begin{tabular}{|c|c|}
5 & $29375-13500 n+1650 n^{2}$ \\
6 & $68256-26460 n+2730 n^{2}$ \\
7 & $140287-47040 n+4200 n^{2}$ \\
8 & $263168-77760 n+6120 n^{2}$ \\
9 & $459999-121500 n+8550 n^{2}$ \\
10 & $760000-181500 n+11550 n^{2}$ \\
11 & $1199231-261360 n+15180 n^{2}$ \\
12 & $1821312-365040 n+19500 n^{2}$ \\
13 & $2678143-496860 n+24570 n^{2}$ \\
14 & $3830624-661500 n+30450 n^{2}$ \\
15 & $5349375-864000 n+37200 n^{2}$ \\
16 & $7315456-1109760 n+44880 n^{2}$ \\
17 & $9821087-1404540 n+53550 n^{2}$ \\
18 & $12970368-1754460 n+63270 n^{2}$ \\
19 & $16879999-2166000 n+74100 n^{2}$ \\
20 & $21680000-2646000 n+86100 n^{2}$ \\
21 & $27514431-3201660 n+99330 n^{2}$ \\
22 & $34542112-3840540 n+113850 n^{2}$ \\
23 & $42937343-4570560 n+129720 n^{2}$ \\
24 & $52890624-5400000 n+147000 n^{2}$ \\
25 & $64609375-6337500 n+165750 n^{2}$ \\
26 & $78318656-7392060 n+186030 n^{2}$ \\
27 & $94261887-8573040 n+207900 n^{2}$ \\
28 & $112701568-9890160 n+231420 n^{2}$ \\
29 & $133919999-11353500 n+256650 n^{2}$ \\
30 & $158220000-12973500 n+283650 n^{2}$ \\
\hline
\end{tabular}

Figure 17. Table for $m=2$, generating function: $\sum_{1 \leq k \leq T} D_{m}(n, k)$ over $T=1,2, \ldots, 30$.

\begin{tabular}{|c|c|}
\hline$t$ & Polynomial $(n)$ \\
\hline 1 & $-125+406 n-420 n^{2}+140 n^{3}$ \\
2 & $-9028+13818 n-7140 n^{2}+1260 n^{3}$ \\
3 & $-110961+115836 n-41160 n^{2}+5040 n^{3}$ \\
4 & $-684176+545860 n-148680 n^{2}+14000 n^{3}$ \\
5 & $-2871325+1858290 n-411180 n^{2}+31500 n^{3}$ \\
6 & $-9402660+5124126 n-955500 n^{2}+61740 n^{3}$ \\
7 & $-25872833+12182968 n-1963920 n^{2}+109760 n^{3}$ \\
8 & $-62572096+25945416 n-3684240 n^{2}+181440 n^{3}$ \\
9 & $-136972701+50745870 n-6439860 n^{2}+283500 n^{3}$ \\
10 & $-276971300+92745730 n-10639860 n^{2}+423500 n^{3}$ \\
11 & $-524988145+160386996 n-16789080 n^{2}+609840 n^{3}$ \\
12 & $-943023888+264896268 n-25498200 n^{2}+851760 n^{3}$ \\
13 & $-1618774781+420839146 n-37493820 n^{2}+1159340 n^{3}$ \\
14 & $-2672907076+646725030 n-53628540 n^{2}+1543500 n^{3}$ \\
15 & $-4267591425+965662320 n-74891040 n^{2}+2016000 n^{3}$ \\
16 & $-6616398080+1406064016 n-102416160 n^{2}+2589440 n^{3}$ \\
17 & $-9995653693+2002403718 n-137494980 n^{2}+3277260 n^{3}$ \\
18 & $-14757360516+2796022026 n-181584900 n^{2}+4093740 n^{3}$ \\
19 & $-21343778801+3835983340 n-236319720 n^{2}+5054000 n^{3}$ \\
20 & $-30303773200+5179983060 n-303519720 n^{2}+6174000 n^{3}$
\end{tabular}




\begin{tabular}{|c|c|c|c|}
21 & $-42311023965+6895305186 n-385201740 n^{2}+7470540 n^{3}$ \\
22 & $-58184203748+9059830318 n-483589260 n^{2}+8961260 n^{3}$ \\
23 & $-78909220801+11763094056 n-601122480 n^{2}+10664640 n^{3}$ \\
24 & $-105663629376+15107395800 n-740468400 n^{2}+12600000 n^{3}$ \\
25 & $-139843308125+19208957950 n-904530900 n^{2}+14787500 n^{3}$ \\
26 & $-183091507300+24199135506 n-1096460820 n^{2}+17248140 n^{3}$ \\
27 & $-237330365553+30225676068 n-1319666040 n^{2}+20003760 n^{3}$ \\
28 & $-304794997136+37454030236 n-1577821560 n^{2}+23077040 n^{3}$ \\
29 & $-388070250301+46068712410 n-1874879580 n^{2}+26491500 n^{3}$ \\
30 & $-490130237700+56274711990 n-2215079580 n^{2}+30271500 n^{3}$ \\
31 & $-614380739585+68298954976 n-2602958400 n^{2}+34442240 n^{3}$ \\
32 & $-764704580608+82391815968 n-3043360320 n^{2}+39029760 n^{3}$ \\
33 & $-945510081021+98828680566 n-3541447140 n^{2}+44060940 n^{3}$ \\
34 & $-1161782683076+117911558170 n-4102708260 n^{2}+49563500 n^{3}$ \\
35 & $-1419139853425+139970745180 n-4732970760 n^{2}+55566000 n^{3}$ \\
36 & $-1723889362320+165366538596 n-5438409480 n^{2}+62097840 n^{3}$ \\
37 & $-2083091040413+194491000018 n-6225557100 n^{2}+69189260 n^{3}$ \\
38 & $-2504622113956+227769770046 n-7101314220 n^{2}+76871340 n^{3}$ \\
39 & $-2997246219201+265663933080 n-8072959440 n^{2}+85176000 n^{3}$ \\
40 & $-3570686196800+308671932520 n-9148159440 n^{2}+94136000 n^{3}$ \\
\hline
\end{tabular}

Figure 18. For $m=3$, generating function: $\sum_{1 \leq k \leq T} D_{m}(n, k)$ over $T=1,2, \ldots, 40$.

\begin{tabular}{|c|c|}
\hline$t$ & Polynomial $(n)$ \\
\hline 1 & $751-2640 n+3780 n^{2}-2520 n^{3}+630 n^{4}$ \\
2 & $162512-325440 n+245700 n^{2}-83160 n^{3}+10710 n^{4}$ \\
3 & $4297023-5837040 n+3001320 n^{2}-695520 n^{3}+61740 n^{4}$ \\
4 & $29586624-47125200 n+18484200 n^{2}-3276000 n^{3}+223020 n^{4}$ \\
5 & $1349845776-949440240 n+253906380 n^{2}-30746520 n^{3}+1433250 n^{4}$ \\
6 & $4981676287-3024769440 n+698619600 n^{2}-73100160 n^{3}+2945880 n^{4}$ \\
7 & $15551330048-8309593440 n+1689523920 n^{2}-155675520 n^{3}+5526360 n^{4}$ \\
8 & $42670773999-20362676400 n+3698370900 n^{2}-304479000 n^{3}+9659790 n^{4}$ \\
9 & $105670786000-45562677600 n+7478370900 n^{2}-556479000 n^{3}+15959790 n^{4}$ \\
10 & $240716895551-94670349840 n+14174871480 n^{2}-962327520 n^{3}+25183620 n^{4}$ \\
11 & $511605381312-184966507440 n+25461891000 n^{2}-1589384160 n^{3}+38247300 n^{4}$ \\
12 & $1025515755823-343092771840 n+43707229020 n^{2}-2525042520 n^{3}+56240730 n^{4}$ \\
13 & $1955262884624-608734803600 n+72168875100 n^{2}-3880359000 n^{3}+80442810 n^{4}$ \\
14 & $3569884005375-1039300430400 n+115225437600 n^{2}-5793984000 n^{3}+112336560 n$ \\
15 & $6275713432576-1715757781440 n+178643314080 n^{2}-8436395520 n^{3}+153624240 n^{4}$ \\
16 & $10670440655087-2749811239440 n+269883324900 n^{2}-12014435160 n^{3}+206242470 n^{4}$ \\
17 & $17613015856848-4292605722240 n+398449531620 n^{2}-16776146520 n^{3}+272377350 n^{4}$ \\
18 & $28312660615999-6545162506800 n+576282961800 n^{2}-23015916000 n^{3}+354479580 n^{4}$ \\
19 & $44440660664000-9770762509200 n+818202961800 n^{2}-31079916000 n^{3}+455279580 n^{4}$ \\
20 & $68269062114351-14309505635040 n+1142398899180 n^{2}-41371850520 n^{3}+577802610 n^{4}$ \\
21 & $102840862500112-20595287515440 n+1570974936300 n^{2}-54359003160 n^{3}+725383890 n^{4}$ \\
22 & $152176783290623-29175447644640 n+2130550596720 n^{2}-70578587520 n^{3}+901683720 n^{4}$ \\
23 & $221524231290624-40733355636000 n+2852919846000 n^{2}-90644400000 n^{3}+1110702600 n^{4}$ \\
24 & $317654602459375-56114215014000 n+3775771408500 n^{2}-115253775000 n^{3}+1356796350 n^{4}$ \\
25 & $449215653223376-76354376660640 n+4943473041780 n^{2}-145194842520 n^{3}+1644691230 n^{4}$ \\
26 &
\end{tabular}




\begin{tabular}{|c|r|r|r|}
27 & $627146261293887-102714466735440 n+6407922490200 n^{2}-181354088160 n^{3}+1979499060 n^{4}$ \\
28 & $865161520339648-136716646589040 n+8229467839320 n^{2}-224724215520 n^{3}+2366732340 n^{4}$ \\
29 & $1180316760605999-180186334891200 n+10477899992700 n^{2}-276412311000 n^{3}+2812319370 n^{4}$ \\
30 & $1593659760714000-235298734894800 n+13233519992700 n^{2}-337648311000 n^{3}+3322619370 n^{4}$ \\
31 & $2130981114417151-304630522458240 n+16588283906880 n^{2}-409793771520 n^{3}+3904437600 n^{4}$ \\
32 & $2823673440038912-391217063149440 n+20647028001600 n^{2}-494350940160 n^{3}+4565040480 n^{4}$ \\
33 & $3709710869661423-498615539455440 n+25528776924420 n^{2}-592972130520 n^{3}+5312170710 n^{4}$ \\
34 & $4834761029884624-630974381822400 n+31368137616900 n^{2}-707469399000 n^{3}+6154062390 n^{4}$ \\
35 & $6253442526125375-793109409951600 n+38316781679400 n^{2}-839824524000 n^{3}+7099456140 n^{4}$ \\
36 & $8030741767978176-990587103477840 n+46545018909480 n^{2}-992199287520 n^{3}+8157614220 n^{4}$ \\
37 & $10243603824112687-1229815433857440 n+56243464735500 n^{2}-1166946059160 n^{3}+9338335650 n^{4}$ \\
38 & $12982712871538448-1518142701993840 n+67624804267020 n^{2}-1366618682520 n^{3}+10651971330 n^{4}$ \\
39 & $16354478705823999-1863964838829600 n+80925655683600 n^{2}-1593983664000 n^{3}+12109439160 n^{4}$ \\
40 & $20483246706016000-2276841638834400 n+96408535683600 n^{2}-1852031664000 n^{3}+13722239160 n^{4}$ \\
\hline
\end{tabular}

Figure 18. For $m=4$, generating function: $\sum_{1 \leq k \leq T} D_{m}(n, k)$ over $T=1,2, \ldots, 40$.

E-mail address: kolosovp94@gmail.com

$U R L$ : https://kolosovpetro.github.io 